\title{
UNC-2 CaV2 Channel Localization at Presynaptic Active Zones Depends on UNC-10/RIM and SYD-2/Liprin- $\alpha$ in Caenorhabditis elegans
}

\author{
Kelly H. Oh, ${ }^{1}$ Mia D. Krout, ${ }^{2}$ Janet E. Richmond, ${ }^{2}$ and ${ }^{\circledR}$ Hongkyun Kim ${ }^{1}$ \\ ${ }^{1}$ Center for Cancer Cell Biology, Immunology, and Infection, Department of Cell Biology and Anatomy, Chicago Medical School, School of Graduate \\ and Postdoctoral Studies, Rosalind Franklin University of Medicine and Science, North Chicago, Illinois 60064, and ${ }^{2}$ Department of Biological \\ Science, University of Illinois at Chicago, Chicago, Illinois 60607
}

Presynaptic active zone proteins couple calcium influx with synaptic vesicle exocytosis. However, the control of presynaptic calcium channel localization by active zone proteins is not completely understood. In a Caenorhabditis elegans (C. elegans) forward genetic screen, we find that UNC-10/RIM (Rab3-interacting molecule) and SYD-2/Liprin- $\alpha$ regulate presynaptic localization of UNC-2, the CaV2 channel ortholog. We further quantitatively analyzed live animals using endogenously GFP-tagged UNC-2 and active zone components. Consistent with the interaction between RIM and CaV2 in mammals, the intensity and number of UNC-2 channel puncta at presynaptic terminals were greatly reduced in unc-10 mutant animals. To understand how SYD-2 regulates presynaptic UNC-2 channel localization, we analyzed presynaptic localization of endogenous SYD-2, UNC-10, RIMB-1/RIM-BP (RIM binding protein), and ELKS-1. Our analysis revealed that although SYD-2 is the most critical for active zone assembly, loss of SYD-2 function does not completely abolish presynaptic localization of UNC-10, RIMB-1, and ELKS-1, suggesting an existence of SYD-2-independent active zone assembly. UNC-2 localization analysis in double and triple mutants of active zone components show that SYD-2 promotes UNC-2 localization by partially controlling UNC-10 localization, and ELKS-1 and RIMB-1 also contribute to UNC-2 channel localization. In addition, we find that core active zone proteins are unequal in their abundance. Although the abundance of UNC-10 at the active zone is comparable to UNC2, SYD-2 and ELKS-1 are twice more and RIMB-1 four times more abundant than UNC-2. Together our data show that UNC-10, SYD-2, RIMB-1, and ELKS-1 control presynaptic UNC-2 channel localization in redundant yet distinct manners.

Key words: active zone; C. elegans; voltage-gated calcium channel

Significance Statement

Precise control of neurotransmission is dependent on the tight coupling of the calcium influx through voltage-gated calcium channels (VGCCs) to the exocytosis machinery at the presynaptic active zones. However, how these VGCCs are tethered to the active zone is incompletely understood. To understand the mechanism of presynaptic VGCC localization, we performed a $C$. elegans forward genetic screen and quantitatively analyzed endogenous active zones and presynaptic VGCCs. In addition to RIM, our study finds that SYD-2/Liprin- $\alpha$ is critical for presynaptic localization of VGCCs. Yet, the loss of SYD-2, a core active zone scaffolding protein, does not completely abolish the presynaptic localization of the VGCC, showing that the active zone is a resilient structure assembled by redundant mechanisms.

Received Jan. 12, 2021; revised Apr. 7, 2021; accepted Apr. 29, 2021.

Author contributions: K.H.O. and H.K. designed research; K.H.O., M.D.K., J.E.R., and H.K. performed research; K.H.O., M.D.K., J.E.R., and H.K. analyzed data; and K.H.O. and H.K. wrote the paper.

This work was supported by the National Institutes of Health National Institute of General Medical Sciences Grant R01-GM-123103 to H.K. and J.E.R. Some strains were provided by the Caenorhabditis Genetics Center, funded by the National Institutes of Health Office of Research Infrastructure Programs (Grant P40 0D010440).

The authors declare no competing financial interests.

Correspondence should be addressed to Hongkyun Kim at hongkyun.kim@rosalindfranklin.edu.

https://doi.org/10.1523/JNEUROSCI.0076-21.2021

Copyright $(2021$ the authors

\section{Introduction}

The active zone of presynaptic terminals is a specialized compartment in which electrical signals are converted to chemical signals. In response to depolarization, voltage-gated calcium channels (VGCCs) deliver extracellular calcium ions, which in turn mediate the fusion of neurotransmitter-filled synaptic vesicles with the presynaptic membrane, resulting in neurotransmitter release and subsequent postsynaptic receptor activation (Südhof, 2012; Dolphin and Lee, 2020). This conversion process requires close coupling of calcium influx with primed synaptic 
vesicles within the active zone. Thus, the precise positioning between docked synaptic vesicles and VGCCs is a critical determinant for the kinetics and efficiency of neurotransmitter release.

The organization of the active zone is structurally and/or functionally supported by a dense network of conserved scaffolding proteins called the cytomatrix at the active zone (CAZ; Jin and Garner, 2008; Ackermann et al., 2015; Emperador-Melero and Kaeser, 2020). The core components of this scaffolding structure include Rab3-interaction protein (RIM), RIM-binding protein (RIM-BP), Liprin- $\alpha$, and glutamine, leucine, lysine, and serine-rich protein (ELKS)/CAZ-associated structural protein (CAST). One intrinsic feature of active zone proteins is their multivalent interactions with other CAZ proteins. Distinct domains of single active zone proteins simultaneously mediate the interactions with other active zone proteins. Because of these redundant multivalent interactions, it is believed that the absence of a single active zone protein, with a few exceptions, does not alter the presynaptic localization of other active zone proteins or the structure of active zones (Acuna et al., 2016; Wang et al., 2016; Kushibiki et al., 2019).

The localization of the P/Q-type VGCC/CaV2.1, a major VGCC found at presynaptic active zones, is regulated by CAZ proteins. The abundance of $\mathrm{CaV} 2.1$ channels correlates with the level of CAZ proteins and the size of active zones (Holderith et al., 2012). Furthermore, the cytoplasmic region of the pore-forming CaV2.1 $\alpha_{1}$ subunit provides binding sites for RIM1/2, RIMBP, and ELKS (Hibino et al., 2002; Fouquet et al., 2009; Kaeser et al., 2011; Kiyonaka et al., 2012; Lübbert et al., 2019). Complete ablation of all RIM proteins in mice reduces the level of CaV2.1 at active zones by $\sim 50 \%$ (Kaeser et al., 2011). In contrast, removal of RIM-BP2 in mice does not reduce the overall levels of $\mathrm{CaV} 2.1$ and $\mathrm{Ca}^{2+}$ influx, but the relative positioning of $\mathrm{CaV} 2.1$ within active zones is altered (Acuna et al., 2015; Grauel et al., 2016). ELKS proteins have also been proposed to influence either $\mathrm{CaV} 2$ activation or levels at active zones but in a synapse-type specific manner (Liu et al., 2014; Held et al., 2016; Dong et al., 2018; Radulovic et al., 2020). In addition to CAZ proteins, neurexins, evolutionarily conserved presynaptic adhesion molecules, incorporate $\mathrm{CaV} 2$ channels at active zone and mediate their precise coupling to release sites (Zhang et al., 2005; Luo et al., 2020; Gomez et al., 2021).

The role of individual active zone proteins in the presynaptic localization of synaptic vesicles, $\mathrm{CaV} 2$ channels, and other CAZ proteins has been assessed in loss-of-function mutants or genetically ablated invertebrate and mouse models. Because this strategy generally employs transgenic presynaptic markers or relies on immunostainings of synaptic proteins, it is often challenging to make a quantitative comparison. In this study, we took advantage of CRISPR/Cas9 genome editing to label endogenous key active zone proteins and CaV2/UNC-2 with GFP and then assessed each of their in vivo synaptic localizations in Caenorhabditis elegans (C. elegans) neurons. We found that the highly conserved RIM ortholog, UNC-10, plays a pivotal role in localizing the $\mathrm{CaV} 2$ channel ortholog, $\mathrm{UNC}-2$, at presynaptic terminals. Although the absence of ELKS-1 or RIMB-1 alone does not cause a significant alteration in presynaptic UNC-2 localization, the absence of both together with UNC-10 further reduces UNC-2 localization. Additionally, we found that SYD-2/Liprin- $\alpha$ is critical for UNC-2 presynaptic localization contributing via two distinct but overlapping mechanisms. SYD-2 maintains UNC-10 localization at presynaptic terminals to indirectly regulate presynaptic UNC-2 localization. SYD-2 also acts on other active zone proteins, including ELKS-1 and RIMB-1, to control presynaptic UNC-2 localization.

\section{Materials and Methods}

Genetic screen for mutants that have an altered UNC-2 localization EMS mutagenized F2 progeny of slo-1(eg142);unc-2(cim114[GFP::unc-2 (L218S)]) animals were screened for $1 \mathrm{~mm}$ aldicarb resistance after $60 \mathrm{~min}$. Based on their movement phenotypes, the confirmed candidate animals were tested for complementation with unc-36(e250), unc-2(e55), calf-1(ky867), and unc-10(md1117). As unc-2(e55) and unc-10(md1117) mutations were on $\mathrm{X}$ chromosome, we used extrachromosomally rescued transgenic animals to perform complementation testing. Select strains were subjected to whole-genome sequencing to identify specific mutation sites.

\section{CRISPR/Cas9-mediated genome editing}

GFP (green fluorescent protein) or mScarlet was inserted into the genome using the coconversion method (Arribere et al., 2014; Cheung et al., 2020). Genome editing was performed by injecting either plasmids expressing guide RNA and Cas9 or a riboprotein mixture of synthesized guide RNA (Integrated DNA Technologies) and custom purified Cas9 protein into the gonad of young adult hermaphrodites. The $5^{\prime}$ and $3^{\prime}$ flanking sequences of genomic target insertion sites ranging from $500 \mathrm{bp}$ to $1 \mathrm{kbp}$ were subcloned to a plasmid containing the GFP or mScarlet coding sequence, and the resulting constructs were used as homologous repair templates.

\section{Aldicarb-induced paralysis assay}

Age-matched adult animals ( $20 \mathrm{~h}$ post-L4) were assayed for their sensitivity to $1 \mathrm{~mm}$ aldicarb. Detailed protocol was published previously (Oh and Kim, 2017).

\section{Electrophysiology}

Electrophysiological recordings from the C. elegans neuromuscular junction were performed as previously described (Richmond, 2006). Briefly, animals were immobilized with Histoacryl glue, and a lateral cuticle incision was made with a glass needle, exposing ventral neuromuscular junctions. Evoked postsynaptic currents were recorded from ventromedial body wall muscles, whole-cell voltage clamped at $-60 \mathrm{mV}$ in response to a $2 \mathrm{~ms}$ depolarizing stimulus applied to the ventral nerve cord, using the following extracellular and intracellular solutions. The extracellular solution consisted of the following (in $\mathrm{mM}$ ): $150 \mathrm{NaCl}, 5 \mathrm{KCl}, 1 \mathrm{CaCl}_{2}, 4$ $\mathrm{MgCl}_{2}, 10$ glucose, 5 sucrose, and 15 HEPES, $\mathrm{pH} \mathrm{7.3,} 340 \mathrm{mOsm}$. The patch pipette was filled with the following (in $\mathrm{mm}$ ): $120 \mathrm{KCl}$, $20 \mathrm{KOH}, 4 \mathrm{MgCl}_{2}, 5 \mathrm{~N}$-tris(Hydroxymethyl)methyl-2-aminoethane-sulfonic acid, $0.25 \mathrm{CaCl}_{2}, 4 \mathrm{Na}_{2} \mathrm{ATP}, 36$ sucrose, and 5 EGTA, pH 7.2, $\sim 315 \mathrm{mOsm}$. Data were acquired using Pulse software (HEKA) run on a Dell computer. Subsequent analysis and graphing were performed using Pulsefit (HEKA), Mini Analysis Program (Synaptosoft), and Igor Pro (Wavemetrics).

\section{Microscopy and image analysis}

Day 1 adult animals (20-24 h post-L4) were immobilized on a $2 \%$ agarose pad with a $6 \mathrm{~mm}$ levamisole solution in M9 buffer. Images were acquired using a $63 \times / 1.4$ numerical aperture on a Zeiss Axio-Observer $\mathrm{Z} 1$ microscope. Images were captured with an $84 \%$ quantum efficiency Zyla 4.2 PLUS (Oxford Instruments; RRID:SCR_017366) using a solidstate Spectra $\mathrm{X}$ light engine (Lumencor) as a light source in the same settings (light intensity and exposure time) for a given GFP transgenic animal. For consistent imaging of the same presynaptic terminals, we imaged an area of the dorsal nerve cord above the posterior gonad arm, which has minimal intestinal autofluorescence. This area consists of GABAergic and cholinergic presynaptic terminals and lacks cell bodies and dendrites. Although we included wild-type controls to ensure that different results obtained in mutants were not because of changes in illumination, the results from different sessions are within the margin of error. The maximum projection was applied to images obtained as a Zstack with a $0.2 \mu \mathrm{m}$ interval. A line-scanning method in MetaMorph Microscopy Automation and Image Analysis Software (RRID:SCR 002368) was used for quantifying the average fluorescence intensity. In each image, a pixel intensity of 282 pixel length $(30 \mu \mathrm{m})$ was measured, 
followed by a subtraction of background intensity from adjacent 282 pixels. From background-subtracted intensity values, peaks with a larger than a threshold value are identified as peaks of clusters. The threshold was arbitrarily set to exclude small background fluctuations, and this same threshold value was applied to all the images of a given protein; one threshold value for all the GFP::UNC-2 images, one value for all the UNC-10, and so on (see Fig. 7, where a different threshold was set because a higher light intensity was used to image GFP::UNC-2 in syd-2 (ok217) mutant background animals). To quantify puncta size, we used Integrative Morphology Analysis (MetaMorph 7.10) to obtain the pixel area of individual puncta.

Microscopic relative copy number quantification of active zone proteins. To compare average and maximum puncta intensities among active zone proteins, we used a Prime 95B camera (Teledyne Photometrics; RRID:SCR_018464) to acquire Z-stack images from the dorsal nerve cord of transgenic animals with endogenously GFP-tagged active zone proteins or CaV2 UNC-2 channels. The high quantum efficiency (95\%) and large pixel size $(11 \mu \mathrm{m})$ of the camera allowed to image all the tested active zone proteins and CaV2/UNC-2 in the same acquisition setting (light intensity and exposure time). To quantify puncta intensity, we first generated a maximum-intensity projection from the Z-stack images, generated a binary mask derived from adaptive thresholding, applied the binary mask to a select dorsal cord region of the maximum-intensity projection image, and then used Integrative Morphology Analysis (MetaMorph 7.10) to get the average and maximum intensities of individual puncta. The final intensity values were obtained by subtracting a background intensity value of an adjacent area. The average and maximum intensities from 10 animals were pooled for analysis.

Thrashing assay. Detailed protocol was described previously (Oh et al., 2020). Age-matched adult animals ( $24 \mathrm{~h}$ post-L4) were placed in $1 \mathrm{ml}$ M9 buffer on an unseeded nematode growth media plate. After acclimation in M9 medium for $5 \mathrm{~min}$, dorsoventral bending movements were recorded for $1 \mathrm{~min}$ using Image Pro 10 (Media Cybernetics). A single thrash was defined as a complete movement cycle through the midpoint and back.

\section{Statistical analysis}

We used GraphPad Prism version 9 (RRID:SCR_002798) to perform statistical analysis. Sample number, $p$ values, and statistical tests used are presented in the legends for Figures 1-10. For aldicarb assay, the Logrank test was used, and Bonferroni correction was applied for multiple comparison. An unpaired $t$ test was used to compare group means for two groups, and an ordinary one-way ANOVA with Tukey's post hoc analysis was used to compare group means for three or more groups. To compare frequency distribution of three or more groups, the KruskalWallis test with Dunn's post hoc analysis was used.

\section{Results}

\section{UNC-10/RIM and SYD-2/Liprin- $\alpha$ are required for presynaptic localization of UNC-2/CaV2}

To understand how endogenous CaV2 channels are localized to presynaptic terminals, we inserted GFP into the amino-terminal region of UNC-2 in the endogenous locus using CRISPR/Cas9 genome editing (Fig. 1A). The resulting animal, cim104[GFP:: unc-2], was healthy and exhibited normal movements indistinguishable from wild-type animals. To analyze the functional integrity of endogenously tagged GFP::UNC-2, we performed a pharmacological assay using the acetylcholinesterase inhibitor aldicarb to detect alterations in C. elegans cholinergic synaptic transmission (Mahoney et al., 2006; Oh and Kim, 2017). Decreased sensitivity to aldicarb-mediated paralysis is indicative of reduced synaptic transmission. GFP-tagged UNC-2 cim104 animals showed aldicarb sensitivity comparable to that of wildtype N2 animals (Fig. 1B). We also determined whether cim104 animals exhibit normal evoked responses at neuromuscular junctions, using patch-clamp electrophysiology and found that evoked responses were unperturbed compared with wild-type animals (Fig. 1C,D). To further ensure that GFP::UNC-2 localizes to presynaptic active zones, we examined UNC-2 colocalization with the presynaptic active zone protein RIMB-1, the ortholog of mammalian RIM binding protein 1 and 2 . The coding sequence of the red fluorescent $\mathrm{mScarlet}$ protein was inserted into the endogenous rimb-1 locus at its initiation codon. UNC-2 and RIMB-1 imaged in the dorsal nerve cord colocalized in all synaptic puncta (Fig. 1E), demonstrating that the genomic tagging of UNC-2 and RIMB-1 does not interfere with previously reported synaptic colocalization (Kurshan et al., 2018).

To understand the molecular mechanism underlying CaV2 channel localization to the presynaptic active zone, we designed a genetic screen. We first generated the cim112 animal, in which an S218L point mutation was introduced into the coding sequence of UNC-2 of the cim104 animal (Fig. 1A). This S218L mutation and the corresponding mutation in human $\mathrm{CaV} 2$ have been shown to enhance calcium channel function, therefore providing a sensitized background for the screen (van den Maagdenberg et al., 2007; Huang et al., 2019). The calcium-activated BK channel ortholog, SLO-1, colocalizes with UNC-2 channels and negatively regulates UNC-2 channels (Oh et al., $2015,2017)$, and thus its absence would further increase UNC-2 (S218L) function. Therefore, we introduced a slo-1 null mutation, eg142, into the cim112 animal. As expected, the combined effects of slo-1(eg142);unc-2(cim112[GFP::unc-2(S218L)]) mutations produced animals that were aldicarb hypersensitive (Fig. $1 F)$. In this background we screened for mutants that showed resistance to the paralyzing effects of aldicarb in F2 progeny of chemically mutagenized slo-1(eg142);unc-2(cim112) double mutant animals (Fig. 1G).

This screen proved highly selective in identifying mutants that disturb unc-2 function. In addition to unc-2 loss or reduction of function mutants, the screen yielded multiple alleles of unc-36 and calf-1, which encode a CaV2 $\alpha_{2} \delta$ subunit ortholog and an ER membrane protein required for UNC-2 trafficking, respectively (Saheki and Bargmann, 2009). Importantly, two unc-10 and one syd-2 mutants were also identified in the screen, although other mutants that have a defect in CAZ have not been identified thus far. Compared with wild-type animals, both unc10(cim55) and syd-2(cim56) animals show significantly decreased GFP-tagged UNC-2 levels in the nerve ring, a collection of synapses derived from over half of the entire neurons (Fig. 1H,I). Based on these results, we more precisely determined how UNC10 and SYD-2 are involved in UNC-2 channel localization at presynaptic terminals.

For quantification of UNC-2 channels at presynaptic terminals, we used the unc-10(md1117) and syd-2(ok217) null mutant strains. In C. elegans as well as Drosophila and mammals, UNC10 and/or SYD-2 homologs are known to interact with their corresponding RIMB-1 and ELKS-1 homologs. Furthermore, mammalian RIM-BP2 and ELKS proteins have been reported to play a role in CaV2 localization in certain contexts (Liu et al., 2014; Acuna et al., 2015; Grauel et al., 2016; Held et al., 2016; Dong et al., 2018). Therefore, we included rimb-1(ce828) and elks-1 (ok2762) mutants in our analysis of UNC-2 channel localization. For quantitative comparison, we determined the fluorescence intensity of GFP-tagged UNC-2 puncta in a select posterior area of the dorsal nerve cord, where cholinergic and GABAergic neurons make discrete en passant synapses with the dorsal body wall muscles (Fig. 2A,B). We found that the number and intensity of 
A

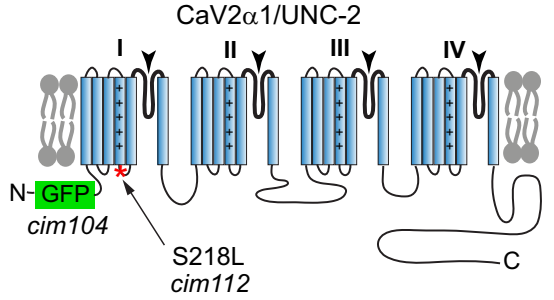

C
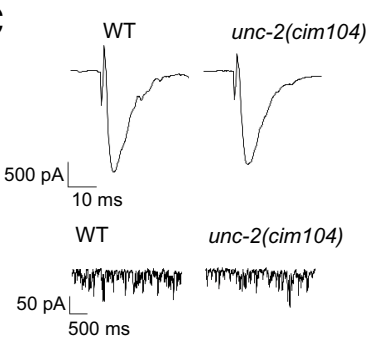

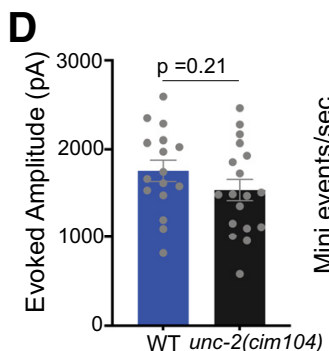

WT unc-2(cim104)
B

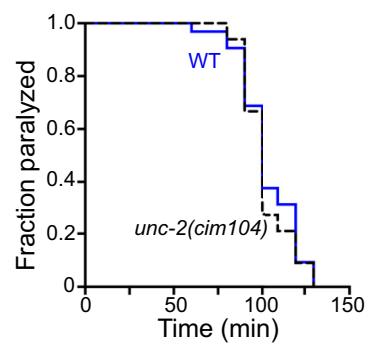

E

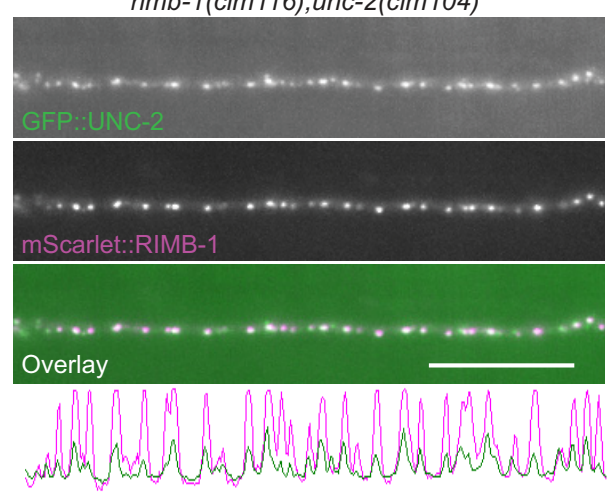

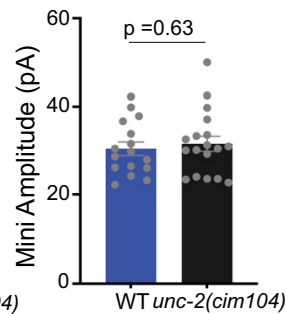

$\mathbf{F}$
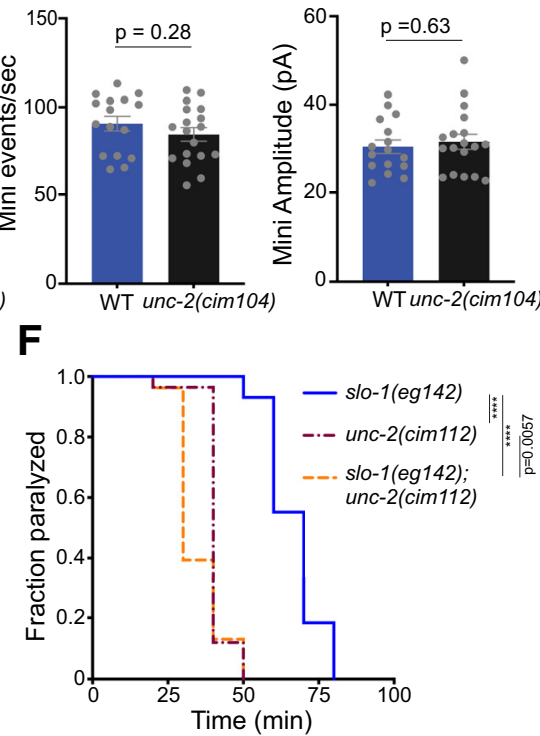

WT unc-2(cim104)
G

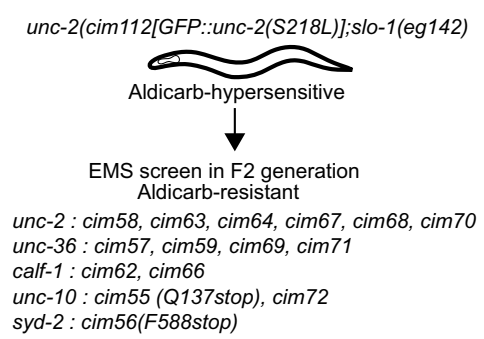

unc-2(cim112[GFP::unc-2(S218L)];slo-1(eg142)

Aldicarb-resistant

unc-2 : $\operatorname{sim} 58, \operatorname{cim} 63, \operatorname{cim} 64, \operatorname{cim} 67, \operatorname{cim} 68, \operatorname{sim} 70$ unc-36 : $\operatorname{cim} 57, \operatorname{cim} 59, \operatorname{cim} 69, \operatorname{cim} 71$ calf-1 : $\operatorname{cim} 62, \operatorname{cim} 66$ unc-10 : $\operatorname{cim} 55$ (Q137stop), $\operatorname{cim} 72$ syd-2 : cim56(F588stop)

\section{H}

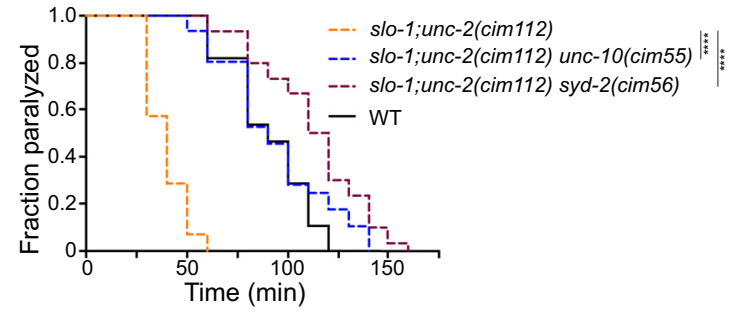

I
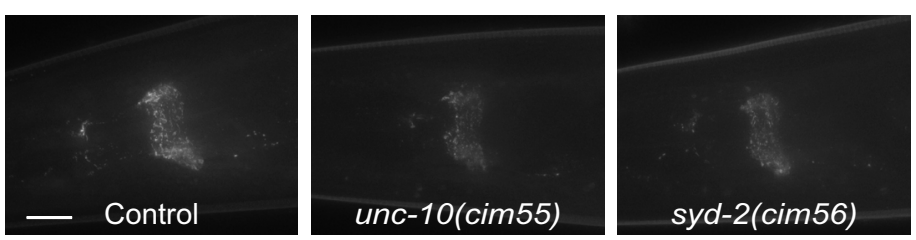

Figure 1. A genetic screen identifies unc-10/RIM and syd-2/Liprin- $\alpha$ as major genes required for UNC-2 localization at presynaptic terminals. $A$, The predicted structure of UNC-2. The relative positions of CRISPR/Cas9-mediated GFP insertion and amino acid substitution (S218L) are denoted. The amino acid position is based on NCBI no. NC_003284.9. B, Aldicarb-mediated paralysis assays show that endogenous GFP tagging of UNC-2 does not alter synaptic function. Fraction of survival on $1 \mathrm{~mm}$ aldicarb plate, $n=32$ for WT, $n=33$ for unc-2(cim 104) Logrank survival test. C, Electrophysiology at the neuromuscular junction shows that endogenous GFP tagging of UNC-2 does not alter synaptic function. Representative trace of patch-clamp electrophysiology at muscle cells at $1 \mathrm{~mm}$ extracellular calcium ions. D, Quantitation of electrophysiological recordings; unc-2(cim104[GFP::unc-2]) animals exhibit a normal evoke response, mini frequency, and mini amplitude in comparison to wild-type animals; two-tailed unpaired $t$ test. E, GFP::UNC-2 and mScarlet::RIMB-1 are colocalized at the dorsal nerve cord. Scale bar, $10 \mu \mathrm{m}$. $\boldsymbol{F}$, Comparison of aldicarb sensitivity of slo-1(eg142), unc-2(cim112[GFP::unc-2S218L]), and slo-1(eg142);unc-2(cim112) animals. The slo-1 gene encodes the calcium-activated BK channel ortholog that negatively regulates CaV2 channels. Log-rank survival test with Bonferroni correction. ${ }^{* * * *} p<0.0001, n=28$ for slo-1(eg142), $n=25$ for unc-2(cim112), $n=23$ for slo-1(eg142); unc-2(cim112). G, A genetic suppressor screen with slo-1(eg142);unc-2(cim112[GFP::unc-2S218L]). The screen with $\sim 2500$ haploid genome size yielded not only genes that affect UNC-2 function and trafficking but also two genes that encode active zone proteins UNC-10/RIM and SYD-2/Liprin- $\alpha$. Because we obtained two independent alleles of calf-1, which encodes a small protein with 177 amino acid residues, we consider that the screen is saturated or close to saturation. $\boldsymbol{H}$, Two presynaptic mutants unc-10(cim55) and syd-2(cim56) suppress aldicarb hypersensitivity of slo-1(eg 142); 4 nc2(cim112[GFP::unc-2S218]) animals. ${ }^{* * * *} p<0.0001, n=17$ for slo-1(eg142);unc-2(cim112), $n=29$ for slo-1(eg142);unc-2(cim112) unc-10(cim55), $n=30$ for slo-1(eg142);unc-2(cim112) syd2(cim56), $n=28$ for WT. Logrank survival test with Bonferroni correction. I, GFP::UNC-2 (S218L) images in the nerve rings of slo-1(eg142); unc-2(cim112), slo-1(eg142);unc-2(cim112) unc-10 (cim55), and slo-1(eg142);unc-2(cim112) syd-2(cim56) mutant animals. The presented images are maximum intensity projection images of a $40 \mathrm{Z}$-stack captured at $0.2 \mu \mathrm{m}$ intervals. Scale bar, $10 \mu \mathrm{m}$. 
A

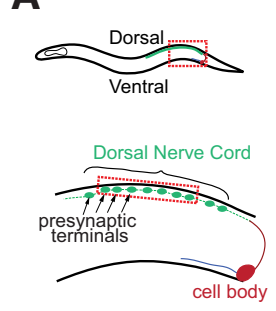

C
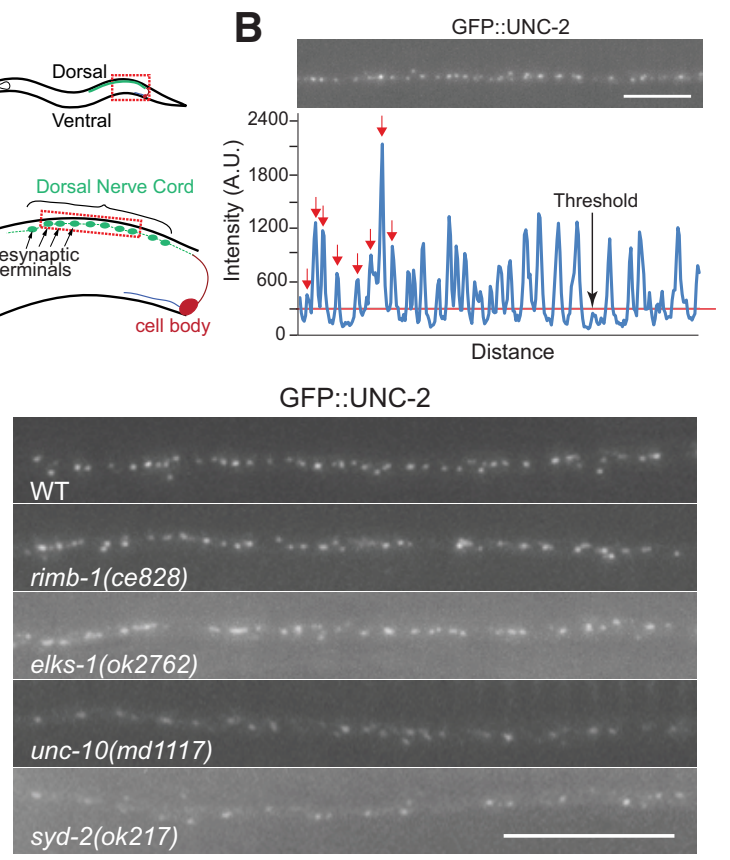

\section{D}
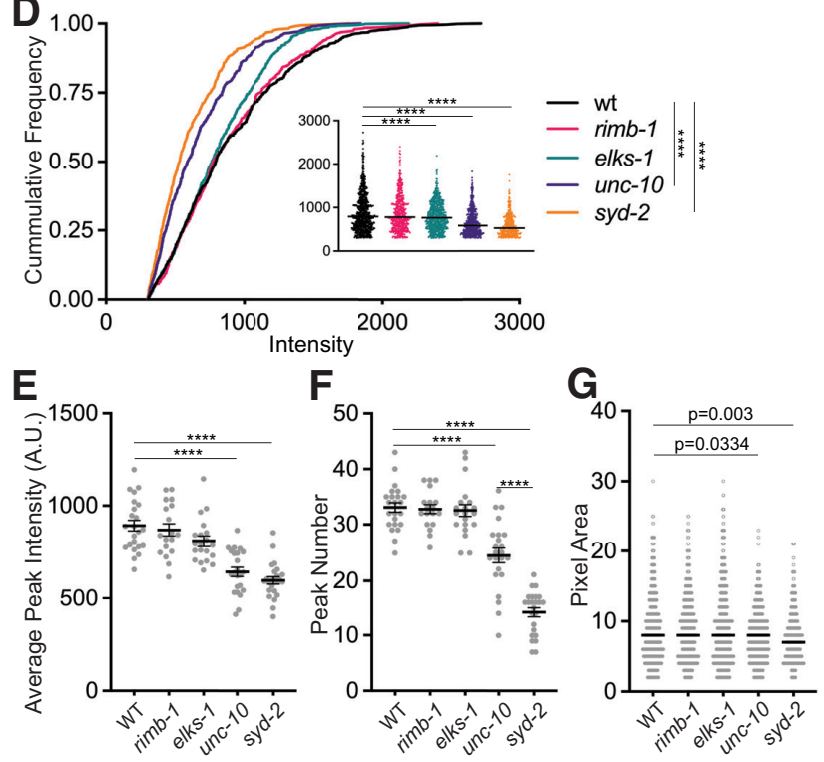

Figure 2. UNC-2 channel localization at presynaptic terminals is regulated by UNC10 and SYD-2. A, Schematics of the imaging strategy of the dorsal nerve cord. The imaged area is part of the dorsal nerve cord positioned above the posterior gonad arm. This area consists of presynaptic terminals of GABAnergic and cholinergic neurons and lacks dendrites or cell bodies. $\boldsymbol{B}$, Peak intensity and peak number were calculated from the linescan method (see Materials and Methods). Arrows indicate examples of peaks identified from the linescan. Scale bar, $5 \mu \mathrm{m}$. C, Endogenous UNC2 puncta in motor neurons of the posterior dorsal nerve cord were reduced in unc-10 and syd-2 mutants but not in elks-1 and rimb-1 mutants. Scale bar, $10 \mu \mathrm{m}$. D, Cumulative frequency distribution of puncta intensity. Individual puncta intensities from a $30 \mu \mathrm{m}$ segment of each animal were pooled $(n=341-761)$. Kruskal-Wallis test with Dunn's post hoc analysis. ${ }^{* * *} p<0.0001$. Inset, Scatter plot of the pooled puncta intensity. The bar represents the median. One-way ANOVA with Tukey's post hoc analysis. $\boldsymbol{E}, \boldsymbol{F}$, Average UNC-2 peak intensity $(\boldsymbol{E})$ and number $(\boldsymbol{F})$ of UNC-2 puncta in a $30 \mu \mathrm{m}$ segment of wild-type (WT), rimb-1, elks-1, unc-10, and syd-2 mutant dorsal nerve cords. Error bars represent SEM. ${ }^{* * *} p<0.0001$. One-way ANOVA with Tukey's post hoc analysis. G, Pixel area of individual puncta. All the puncta from a $30 \mu \mathrm{m}$ segment of each animals were pooled $(n=405-716)$. The bar represents the median. ${ }^{* * *} p<0.0001$. One-way ANOVA with Tukey's post hoc analysis.
GFP::UNC-2 puncta were greatly reduced in unc-10(md1117) compared with wild-type animals (Fig. 2C-F). This finding is consistent with a previous study from conditional knock-out mice of all RIM isoforms (Kaeser et al., 2011), and syd-2(ok217) mutants showed an even more pronounced reduction in UNC-2 puncta number and intensity than unc-10(md1117) mutants. Importantly, the reduced intensities of presynaptic UNC-2 channels in unc-10 and syd-2 mutants are not because of diffuse localization of UNC-2 channels at presynaptic terminals because pixel areas of the puncta were also reduced in $u n c-10$ and syd-2 mutants (Fig. 2G). In contrast, rimb-1(ce828) and elks-1(ok2762) mutants were comparable to wild-type animals in number and intensity of UNC-2 puncta.

\section{SYD-2 plays a prominent role in presynaptic localization of UNC-10, RIMB-1, and ELKS-1}

The results above demonstrate that SYD-2 and UNC-10 affect UNC-2 puncta to differing degrees. SYD-2 has been reported to act as a core active zone protein in C. elegans, and prior studies have demonstrated interactions with RIM proteins as well as other CAZ components including RIM-BP and ELKS (Ohtsuka et al., 2002; Wang et al., 2002; Ko et al., 2003; Dai et al., 2006; Kaeser et al., 2011; Petzoldt et al., 2020). Therefore, we considered the possibility that the effects of the syd-2 mutation on UNC-2 could be because of synaptic alterations of each of these binding partners. To test this possibility, we generated endogenously GFP-tagged strains of UNC-10, RIMB-1, and ELKS-1, as well as SYD-2 using CRISPR/Cas9 genome editing to examine the interdependence of these active zone proteins for synaptic localization. Unlike synaptic vesicle markers that show faint signals along the axon while enriched within presynaptic terminals (Oh et al., 2015), these GFP-tagged active zone proteins showed clearer, discrete puncta. One limitation of our analysis on the interdependent relationship among active zone proteins is that certain rare puncta may not be localized to active zones because some of the active zone proteins, such as SYD-2/Liprin- $\alpha$ and ELKS-1, were also observed at nonsynaptic sites (Wagner et al., 2009; Spangler et al., 2011; Zürner et al., 2011; Nyitrai et al., 2020).

We first compared the peak intensities and numbers of UNC10 puncta in wild-type, rimb-1(ce828), elks-1(ok2762), and syd-2 (ok217) animals (Fig. 3A-D). The peak intensity and number of presynaptic UNC-10 puncta were reduced in syd-2(ok217) mutant animals when compared with wild-type animals, but negligibly different in rimb-1(ce828) or elks-1(ok2762) mutant animals. Having observed a reduction in UNC-10 puncta in syd-2(ok217) mutant animals, we then performed the reciprocal experiments to determine whether SYD-2 presynaptic clusters are altered in unc-10(md1117) mutants as well as in rimb-1(ce828) or elks-1 (ok2762) mutants (Fig. 3E-H). The peak intensities of SYD-2 puncta were minimally affected in all three mutants, confirming that SYD-2 plays a prominent role in presynaptic CAZ organization (Zhen and Jin, 1999; Dai et al., 2006; Patel et al., 2006). These results together indicate that the reduction of UNC-2 puncta in syd-2(ok217) mutants can be partially explained by the role of SYD-2 in UNC-10 presynaptic localization (Fig. 3). Because the syd-2 mutation did not completely abolish presynaptic UNC-10 localization, UNC-2 localization could be supported by the remaining UNC-10 proteins at presynaptic terminals. In this case, we predicted that UNC-2 localization would be more severely affected in $u n c-10$ (md1117) mutant animals than syd-2 (ok217) mutant animals. In contrast to this prediction, we observed that syd-2(ok217) mutant animals exhibit a more severe 
A

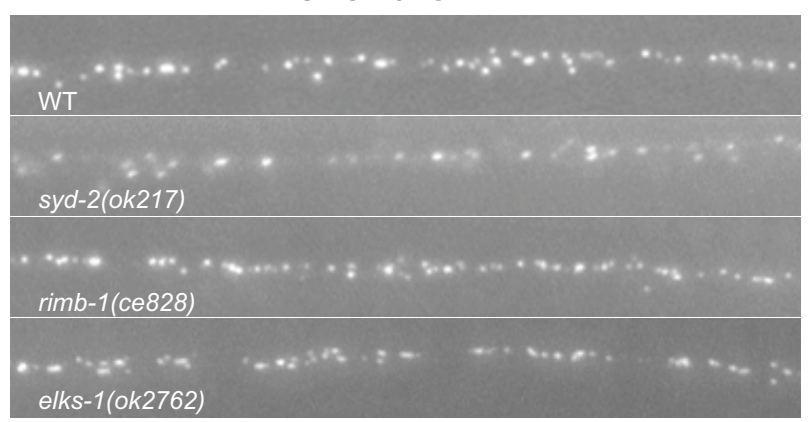

B

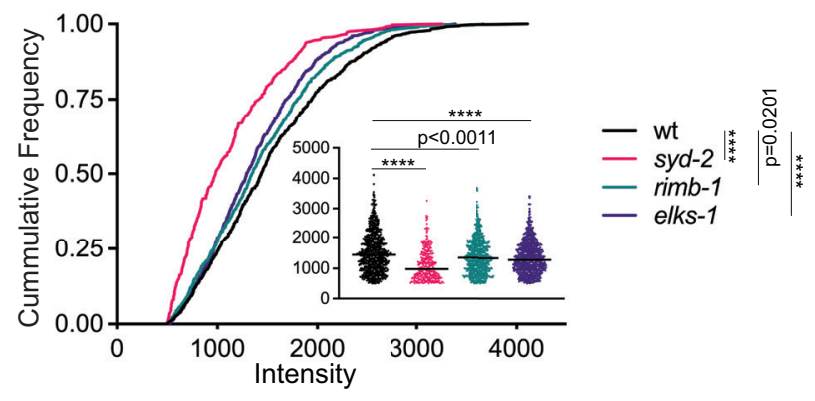

C

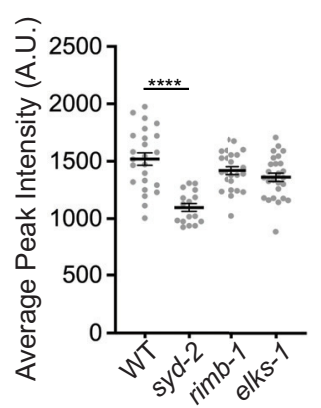

UNC-10::GFP

D

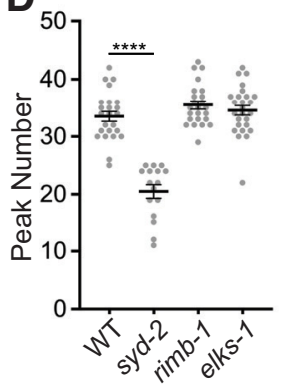

E

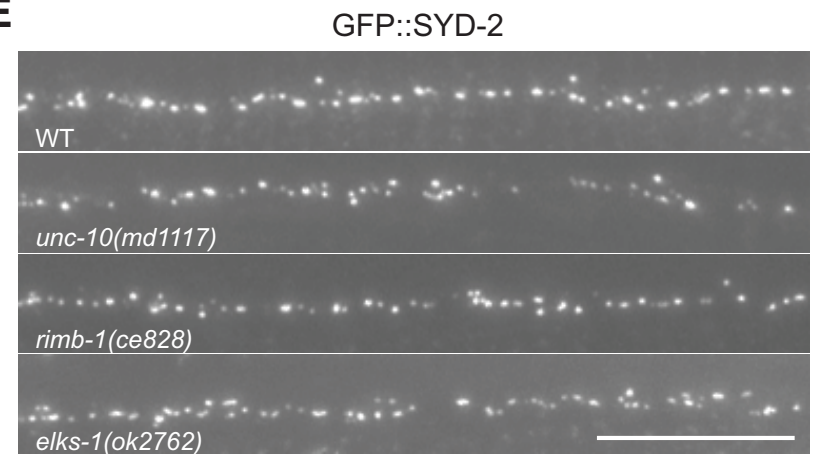

$\mathbf{F}$

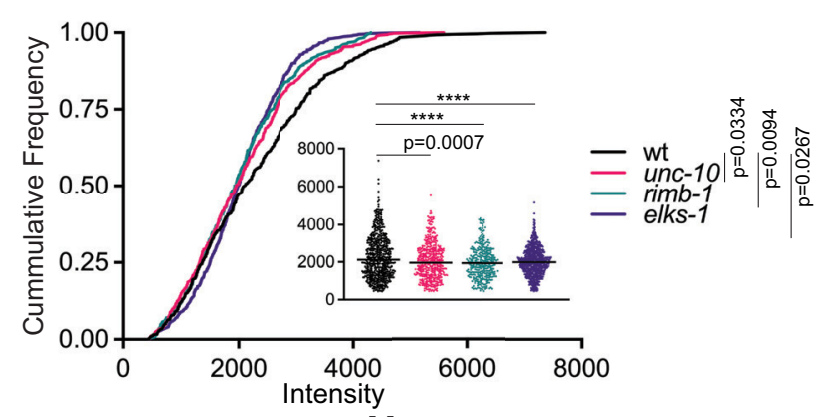

G

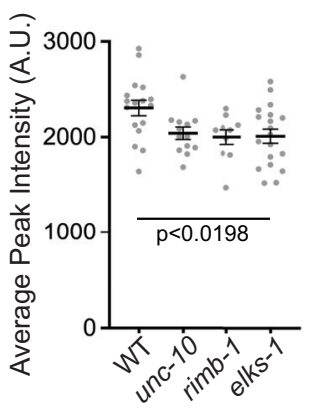

$\mathrm{H}$

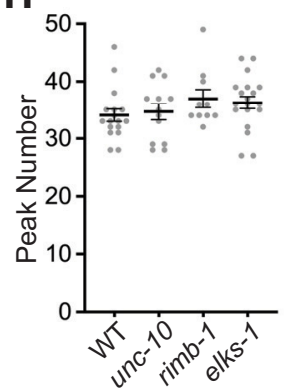

Figure 3. Presynaptic UNC-10 and SYD-2 localizations are mediated by multiple redundant interactions. $A$, UNC-10 puncta at presynaptic terminals are reduced, but not eliminated, in syd-2 mutant animals. Endogenous UNC-10 localization in motor neurons of the posterior dorsal nerve cord is reduced in syd-2 mutants but not in elks-1 and rimb-1 mutants. Scale bar, $10 \mu \mathrm{m}$. $\boldsymbol{B}$, Cumulative frequency distribution of puncta intensity. Individual puncta intensities from a $30 \mu \mathrm{m}$ segment of each animal were pooled $(n=328-899)$. Kruskal-Wallis test with Dunn's post hoc analysis. ${ }^{* * *} p<0.0001$. Inset, Scatter plot of the pooled puncta intensity. The bar represents the median. One-way ANOVA with Tukey's post hoc analysis. C, Average UNC-10 peak intensity in WT, rimb-1, elks-1, and syd-2 mutant animals. ${ }^{* * *} p<0.0001$. One-way ANOVA with Tukey's post hoc analysis. $\boldsymbol{D}$, The number of UNC-10 puncta in the dorsal nerve cords (per a $30 \mu \mathrm{m}$ segment) of wild-type (WT), rimb-1, elks-1, and syd-2 mutant animals. ${ }^{* * *} p<0.0001$. One-way ANOVA with Tukey's post hoc analysis. E, Endogenous SYD-2 localization in the posterior dorsal nerve cords of unc-10, elks-1, rimb-1 mutants. Scale bar, $10 \mu \mathrm{m}$. $\boldsymbol{F}$, Cumulative frequency distribution of puncta intensity. Individual puncta intensities from a $30 \mu \mathrm{m}$ segment of each animal were pooled $(n=370-690)$. Kruskal-Wallis test with Dunn's post hoc analysis. ${ }^{* * *} p<0.0001$. Inset, Scatter plot of the pooled puncta intensity. The bar represents the median. One-way ANOVA with Tukey's post hoc analysis. G, Average SYD-2 peak intensity in wild-type (WT), rimb-1, elks-1, and unc-10 mutant animals. $\boldsymbol{H}$, The number of SYD-2 puncta in the dorsal nerve cords (per a $30 \mu \mathrm{m}$ segment) of wild-type (WT), rimb-1, elks-1, and unc-10 mutant animals. Error bars represent SEM. One-way ANOVA with Tukey's post hoc analysis.

defect in UNC-2 localization than unc-10(md1117) mutant animals (Fig. $2 C-G$ ). Thus, it is likely that SYD-2 provides an additional mechanism for UNC-2 localization, independent of UNC10 localization.

Given that SYD-2 associates with multiple CAZ proteins, we next explored the possibility that in addition to UNC-10, SYD-2 localizes UNC-2 channels to presynaptic terminals through the interaction with RIMB-1 and EKLS-1. First, we examined whether RIMB-1 and ELKS-1 localization are dependent on SYD-2. Although the intensities of RIMB-1 puncta are reduced in unc-10(md1117), elks-1(ok2762), and syd-2(ok217) mutant animals, the extent of the reduction was most prominent in $s y d-2$ (ok217) mutant animals (Fig. 4A-C). In contrast to RIMB-1 puncta intensities, the number of RIMB-1 puncta was only affected in syd-2(ok217) mutants and was accompanied by diffuse intrasynaptic RIMB-1 protein expression (Fig. 4D). Likewise, the GFP intensities of ELKS-1 puncta were also reduced in unc-10(md1117), rimb-1(ce828), and syd-2(ok217) mutant animals (Fig. $4 A-G$ ), with a reduction in puncta number only apparent in syd-2(ok217) mutant animals (Fig. 4H). These results indicate that SYD-2 plays a prominent role in recruiting or stabilizing UNC-10, RIMB-1, and ELKS-1 at presynaptic active zones, whereas these three proteins also contribute to active zone assembly or stabilization.

\section{Stoichiometry of UNC-2, UNC-10, RIMB-1, ELKS-1, and SYD-2}

In imaging endogenously labeled CAZ proteins, we noticed that relative presynaptic GFP fluorescence intensities were markedly different for the components examined. To quantify these relative levels, we imaged using a camera with a higher dynamic range (Fig. 5). We found that the average and maximum intensities of UNC-2/CaV2and UNC-10/RIM puncta were comparable at presynaptic terminals. In contrast, the average and 
A

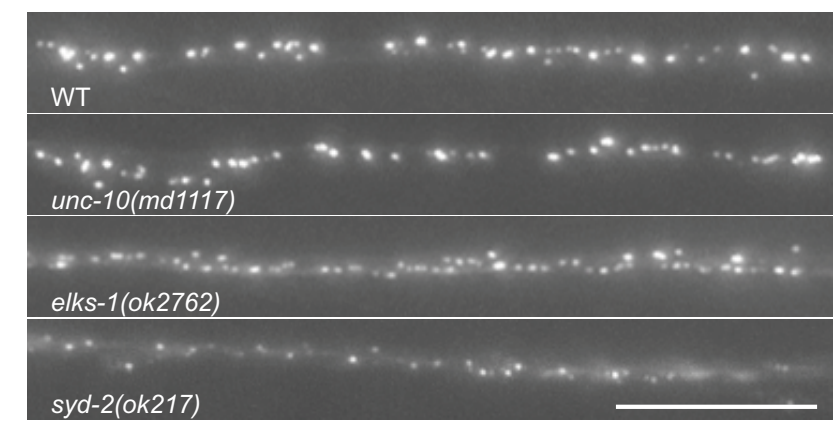

B

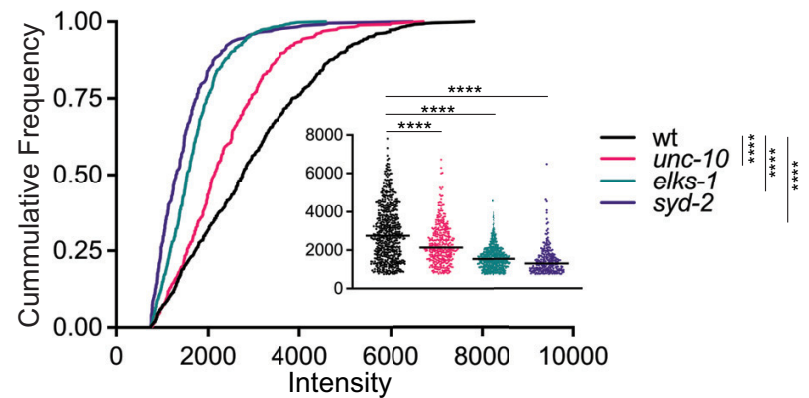

C

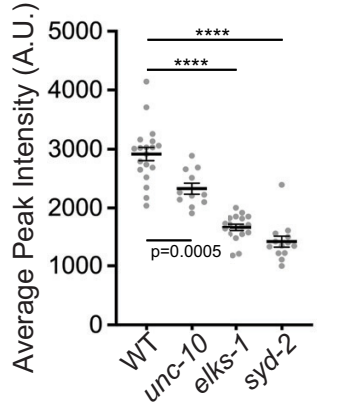

GFP::RIMB-1

D

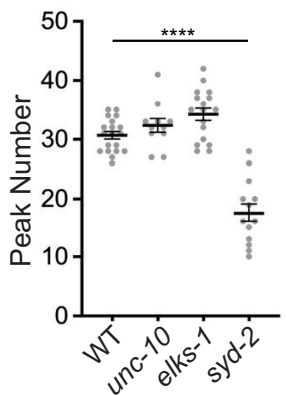

E

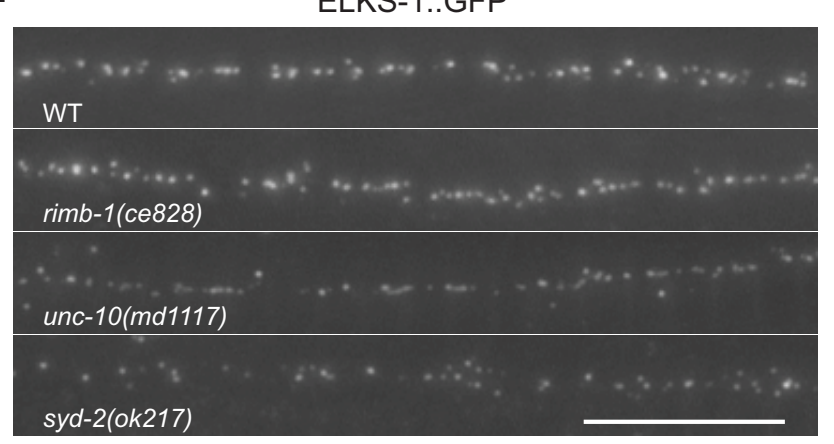

F

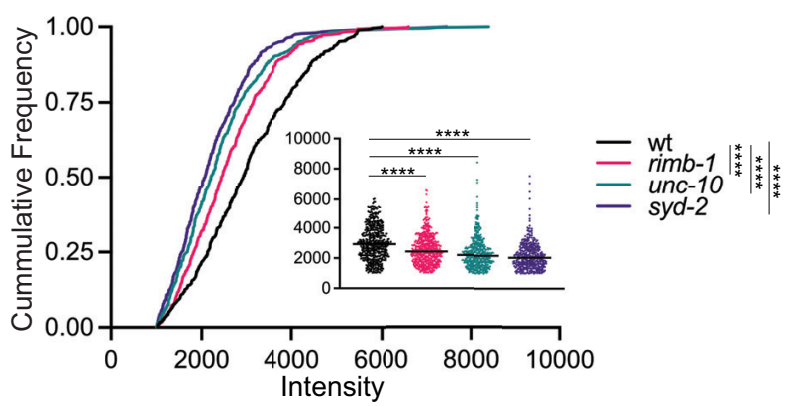

G

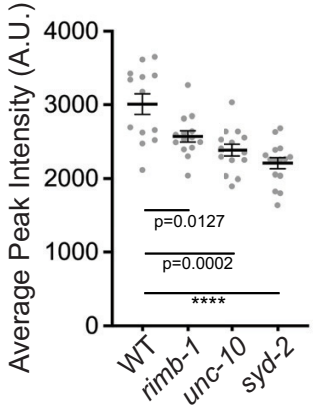

$\mathrm{H}$

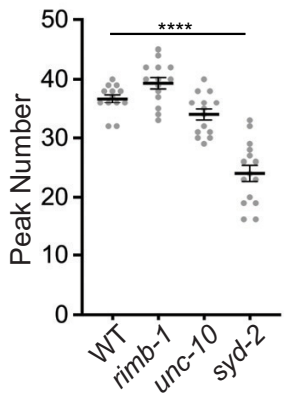

Figure 4. The localization of RIMB-1 and ELKS-1 at presynaptic terminals is partially dependent on SYD-2. $A$, The number and intensity of RIMB-1 puncta are reduced, but not eliminated, in syd-2 mutant animals, and UNC-10 and ELKS-1 are required for the normal level of puncta intensity. Scale bar, $10 \mu \mathrm{m}$. $\boldsymbol{B}$, Cumulative frequency distribution of puncta intensity. Individual puncta intensities from a $30 \mu \mathrm{m}$ segment of each animal were pooled $(n=229-583)$. Kruskal-Wallis test with Dunn's post hoc analysis. ${ }^{* * * *} p<$ 0.0001. Inset, Scatter plot of the pooled puncta intensity. The bar represents the median. One-way ANOVA with Tukey's post hoc analysis. C, Average RIMB-1 peak intensity in wild-type (WT), rimb-1, elks-1, and syd-2 mutant animals. D, The number of RIMB-1 puncta in the dorsal nerve cords (per $30 \mu \mathrm{m}$ ) of wild-type (WT), unc-10, elks-1, and syd-2 mutant animals. $\boldsymbol{E}$, Endogenous ELKS-1 localization in motor neurons of the posterior dorsal nerve cord largely depends on SYD-2. Scale bar, $10 \mu \mathrm{m}$. $\boldsymbol{F}$, Cumulative frequency distribution of puncta intensity. Individual puncta intensities from a $30 \mu \mathrm{m}$ segment of each animal were pooled $(n=361-551)$. Kruskal-Wallis test with Dunn's post hoc analysis. ${ }^{* * * *} p<0.0001$ Inset, Scatter plot of the pooled puncta intensity. The bar represents the median. One-way ANOVA with Tukey's post hoc analysis. G, Average ELKS-1 peak intensity in wild-type (WT), rimb-1, elks-1, and unc-10 mutant animals. $\boldsymbol{H}$, The number of ELKS-1 puncta in the dorsal nerve cords (per $30 \mu \mathrm{m}$ ) of wild-type (WT), rimb-1, elks-1, and unc-10 mutant animals. Error bars represent SEM **** $<0.0001$. One-way ANOVA with Tukey's post hoc analysis.

maximum intensities of presynaptic SYD-2 and ELKS-1 puncta were significantly higher than UNC-2 and UNC-10 puncta by a factor of 2. Whether this twofold increase in SYD-2 and ELKS-1 label intensity relates to previously reported observations that SYD-2 forms homodimers at presynaptic terminals (Taru and Jin, 2011) and physically interacts with ELKS-1 (Deken et al., 2005) remains to be tested. Unexpectedly, RIMB-1 is four times more enriched than UNC-2 or UNC-10 at synapses. The stoichiometry of presynaptic CaV2 and CAZ proteins may reflect differences in copy number because of homodimerization and multimeric assembly of some components.

\section{RIMB-1 and ELKS-1 contribute to synaptic localization of the UNC-2/CaV channel}

RIM, RIM-BP, and ELKS have been previously shown to interact with $\mathrm{CaV} 2$ channels, whereas SYD-2/Liprin- $\alpha$ has not been implicated in CaV2 channel localization. It is possible that SYD2 can directly act on UNC-10, and UNC-10 subsequently recruits RIMB-1 or ELKS-1 to localize UNC-2 channels to presynaptic terminals. Alternatively, SYD-2 may act on RIMB-1 or ELKS-1 independently of UNC-10 to regulate UNC-2 channel localization. To test these possibilities (Fig. 6), we first sought to understand the relationship between UNC-10 with RIMB-1 and ELKS1 by comparing GFP::UNC-2 puncta in unc-10(md1117) single, double, and triple mutants with rimb-1(ce828) and elks-1 (ok2762) (Fig. 6). Although the absence of ELKS-1 did not further alter UNC-2 puncta, the absence of RIMB-1 moderately reduced the peak intensity of UNC-2 puncta of unc-10(md1117) mutant animals without significantly changing the overall number of UNC-2 puncta (Fig. $6 B-D$ ). These results indicate that RIMB-1, but not ELKS-1, contributes to UNC-2 localization when UNC-10 is absent. Interestingly, unc-10(md1117);rimb-1 
A

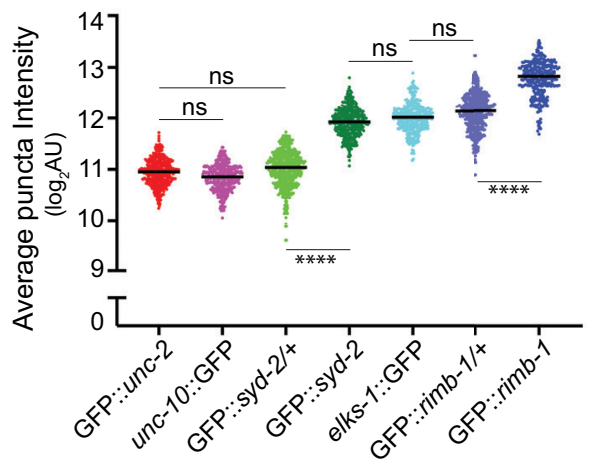

B

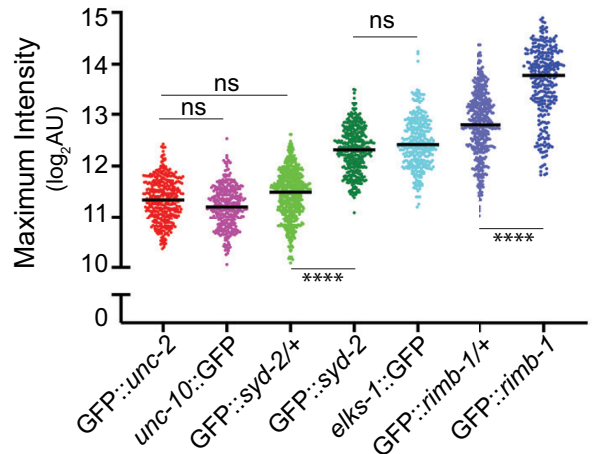

C

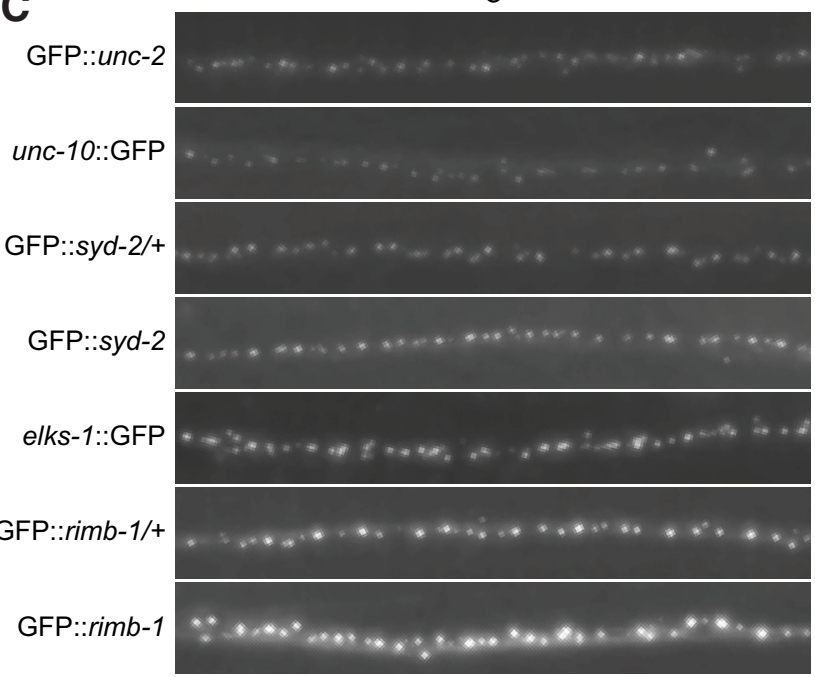

Figure 5. Copy number analysis of UNC-2/CaV2 and cytomatrix proteins at the presynaptic terminals indicates at least three distinct groups. The relative levels of endogenously GFPtagged UNC-2, UNC-10, SYD-2, ELKS-1, and RIMB-1 at the presynaptic terminals are determined in the dorsal nerve cord of animals. To evaluate the relative intensity of GFP puncta, heterozygous GFP::syd-2/+ and GFP::rimb-1/+ animals were also included in the analysis. $A$, The average intensity of UNC-2, UNC-10, SYD-2/+, SYD-2, ELKS-1, RIMB-1/+, and RIMB1 puncta. The data do not pass a normality test, and Kruskal-Wallis test with Dunn's post hoc was used for statistical analysis. $\left.{ }^{* * *} p<0.0001\right)$. $\boldsymbol{B}$, The maximum intensity of UNC-2, UNC-10, SYD-2/+, SYD-2, ELKS-1, RIMB-1/+, and RIMB-1 puncta. The number of puncta ranges approximately from 300 to 600 , as all of the focused puncta within the visual field were pooled from 10 independent animals. ${ }^{* * *} p<0.0001$, Kruskal-Wallis test with Dunn's post hoc analysis. C, The representative images of UNC-2, UNC-10, SYD-2/+, SYD-2, ELKS-1, RIMB-1/+, and RIMB-1 puncta. Images were scaled to the same dynamic range to visualize puncta. ns, not significant.

(ce828);elks-1(ok2762) triple mutant animals exhibit a further reduction in both number and peak intensity of UNC-2 puncta compared with the unc-10(md1117);rimb-1(ce828) double mutant animals. Thus, RIMB-1 and ELKS-1 also contribute to UNC-2 localization, although UNC-10 plays the predominant role.
A

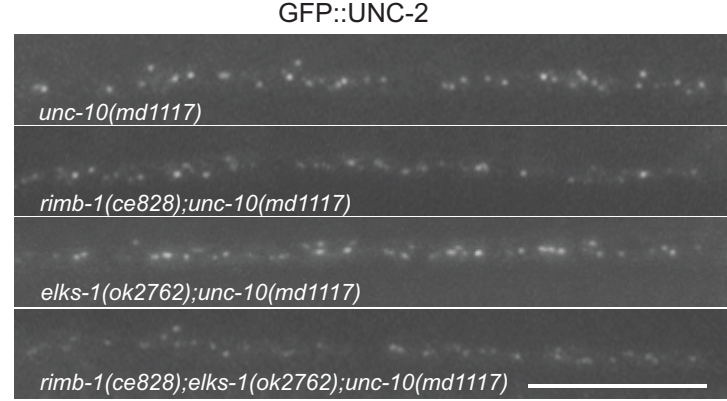

B
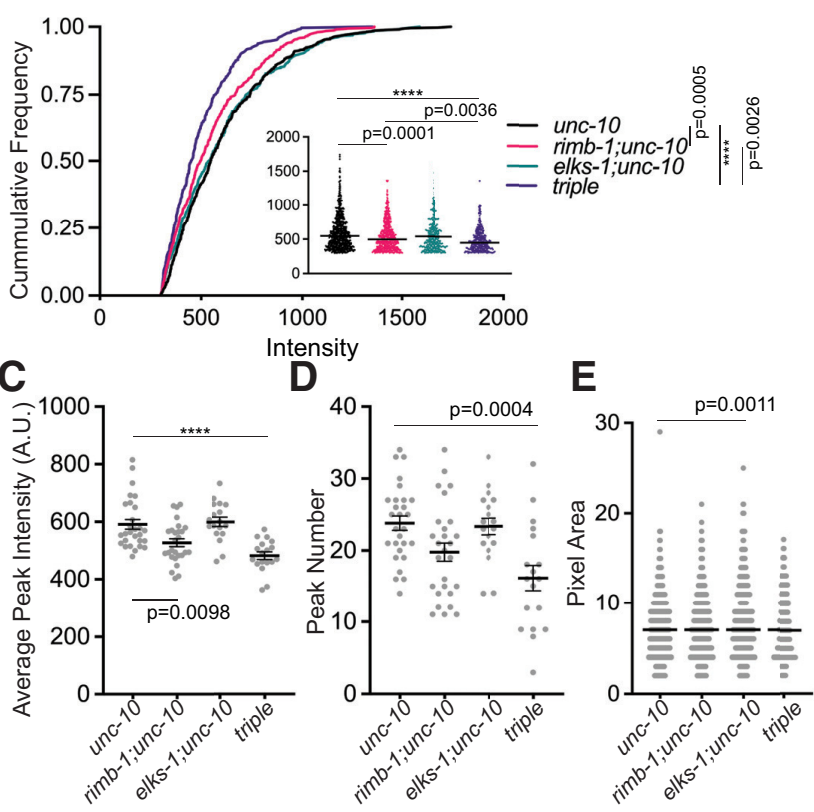

Figure 6. RIMB-1 and ELKS-1 contribute to UNC-2 puncta formation. $A$, Endogenous UNC2 localization in motor neurons of the posterior dorsal nerve cord is further reduced in rimb1; unc-10 double and rimb-1;elks-1; unc-10 triple mutants. Scale bar, $10 \mu \mathrm{m}$. B, Cumulative frequency distribution of puncta intensity. Individual puncta intensities in a $30 \mu \mathrm{m}$ segment of each animal were pooled ( $n=290-667)$. Kruskal-Wallis test with Dunn's post hoc analysis. $* * * * p<0.0001$. Inset, Scatter plot of the pooled puncta intensity. The bar represents the median. ANOVA with Tukey's post hoc analysis. C, Average UNC-2 peak intensity in unc10, rimb-1; unc-10, elks-1; unc-10, and rimb-1; elks-1; unc-10 mutant animals. One-way ANOVA with Tukey's post hoc analysis. $\boldsymbol{D}$, The number of UNC-2 puncta in the dorsal nerve cords (per $30 \mu \mathrm{m}$ ) of unc-10, rimb-1; unc-10, elks-1; unc-10, and rimb-1;elks-1; unc-10 mutant animals. $\boldsymbol{E}$, Pixel area of individual puncta. All the puncta from a $30 \mu \mathrm{m}$ segment of each animal were pooled $(n=274-639)$. The bar represents the median. One-way ANOVA with Tukey's post hoc analysis.

\section{UNC-2/CaV is localized at the synapse by both SYD-2- dependent and SYD-2-independent mechanisms}

Next, we investigated how SYD-2 regulates UNC-2 channel localization at presynaptic terminals in relation to UNC-10, ELKS-1, and RIMB-1. Although the localization of UNC-10, RIMB-1, and ELKS-1 depends largely on SYD-2, all three proteins show residual localization in the absence of SYD-2. To determine whether the remaining GFP::UNC-2 in syd-2 mutants is because of the residual UNC-10, RIMB-1, and ELKS-1, we compared UNC-2 puncta in unc-10( $m$ d1117) syd-2(ok217), elks1(ok2762);syd-2(ok217), and rimb-1(ce828);syd-2(ok217) double mutant animals (Fig. 7). Compared with syd-2(ok217) single mutant animals, presynaptic UNC-2 was further reduced in unc-10 (md1117), syd-2(ok217), and elks-1(ok2762);syd-2(ok217) mutant but not in rimb-1(ce828);syd-2(ok217) mutant. This suggests 
A

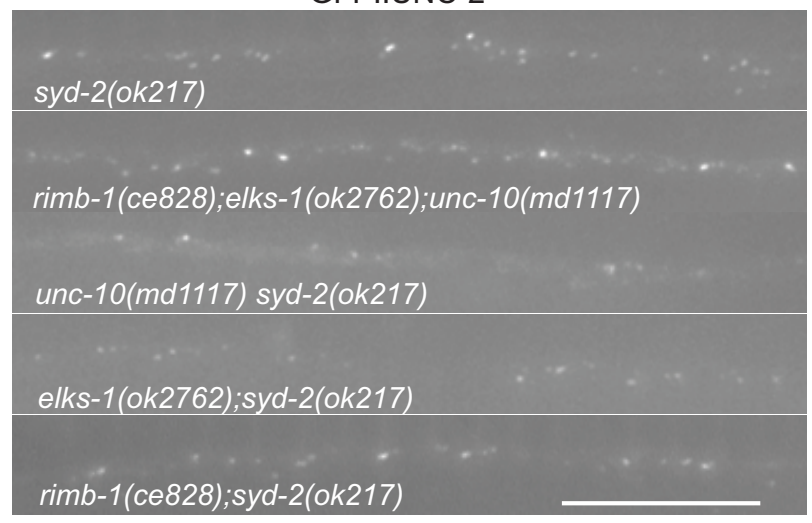

C

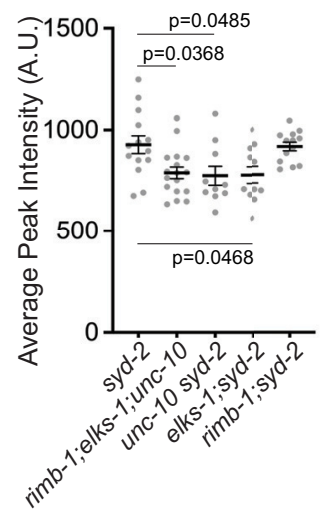

D

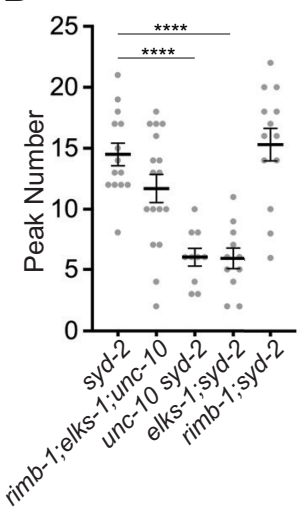

B

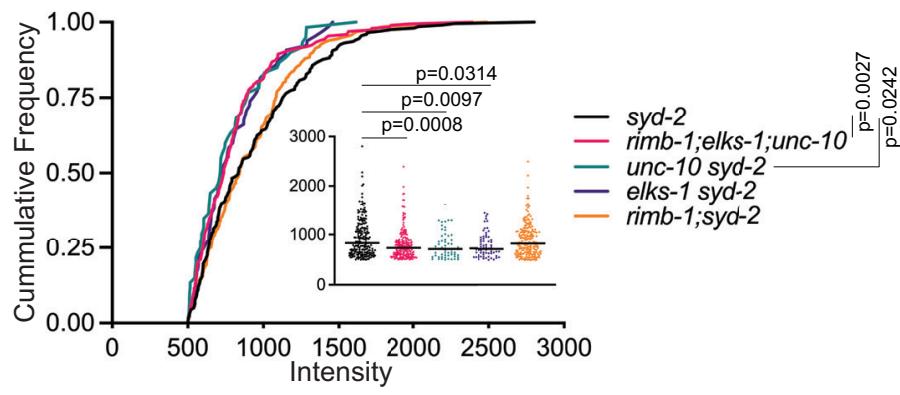

$\mathbf{F}$

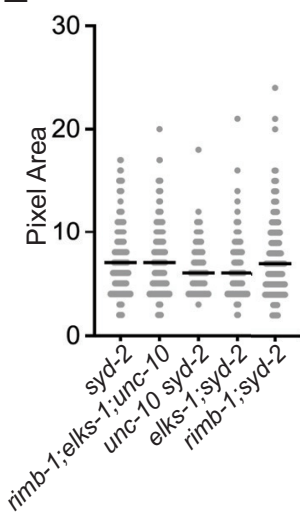

GFP::UNC-2

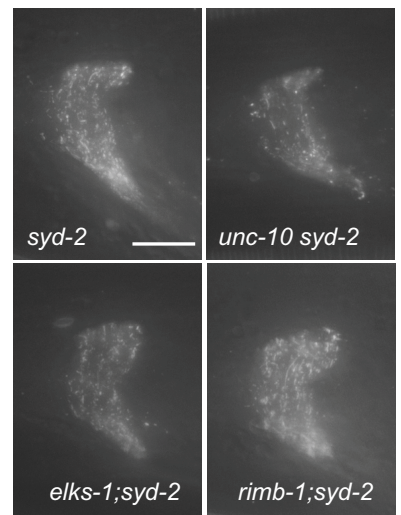

Figure 7. UNC-2 localization is dependent on both SYD-2-dependent and SYD-2-independent mechanisms. $A$, Endogenous UNC-2 localization in motor neurons of the posterior dorsal nerve cord is further reduced in unc-10 syd-2 and elsk-1;syd-2 double mutants. Scale bar, $10 \mu \mathrm{m}$. B. Cumulative frequency distribution of puncta intensity. Individual puncta intensities from a $30 \mu \mathrm{m}$ segment of each animal is pooled $(\mathrm{n}=60-203)$. Kruskal-Wallis test with Dunn's post hoc analysis. ${ }^{* * *} p<0.0001$ Inset, Scatter plot of the pooled puncta intensity. The bar represents the median. ANOVA with Tukey's post hoc analysis. C, Average UNC-2 peak intensity in syd-2, rimb-1; elks-1;unc-10, unc-10 syd-2, elks-1;5yd-2, rimb-1;syd-2 mutant animals. Error bars represent SEM **** $<0.0001$. One-way ANOVA with Tukey's post hoc analysis. D, The number of UNC-2 puncta in the dorsal nerve cords (per $30 \mu \mathrm{m}$ ) of syd-2, rimb-1; elks-1; unc-10, unc-10 syd-2, elks1;syd-2, rimb-1;syd-2 mutant animals. Error bars represent SEM. ${ }^{* * *} p<0.0001$. One-way ANOVA with Tukey's post hoc analysis. $E$, Pixel area of individual puncta. All the puncta from each animal were pooled $(n=69-231)$. The bar represents the median. ANOVA with Tukey's post hoc analysis. $F$, GFP::UNC-2 images in the nerve rings of syd-2, unc-10 syd-2, elks-1;syd-2, and rimb-1;;yd-2 mutant animals. The images are maximum intensity projection images of a 45 Z-stack captured at $0.2 \mu \mathrm{m}$ intervals. A reduction of UNC-2 in unc-10 syd-2 and elks-1;5yd-2 mutant animals is prominent compared with syd-2 and rimb-1;syd-2 mutant animals. Scale bar, $10 \mu \mathrm{m}$.

that SYD-2-independent UNC-10 and ELKS-1 at active zones contribute to UNC-2 localization, although the contribution of RIMB-1 is largely dependent on SYD-2. Next, we examined whether the three proteins, UNC-10, RIMB-1, and ELKS-1, together can account for presynaptic UNC-2 localization. Surprisingly, UNC-2 puncta are still present in rimb-1(ce828); elks-1(ok2762);unc-10(md1117) triple mutants, although the number and intensity of the puncta are slightly lower than in syd-2(ok217) mutants (Fig. 7). This indicates that SYD-2 can localize UNC-2 either through a direct interaction or through a protein other than UNC-10, RIMB-1, or ELKS-1.

\section{Presynaptic UNC-2/CaV2 channel levels correlate with the} mobility profile for $u n c-10$, syd-2, elks-1, and rimb-1 mutant animals

Next, we examined whether UNC-2 channel levels in active zone mutant animals correlate with their mobility using a thrashing assay (Fig. 8). In a liquid medium, C. elegans exhibits dorsoventral swimming (thrashing) behavior, and the measurement of its frequency is a well-established method for assessing mobility (Pierce-Shimomura et al., 2008). The unc-10(md1117) mutants exhibited a reduced thrashing frequency compared with wild-type animals. The rimb-1(ce828);unc-10(md1117)

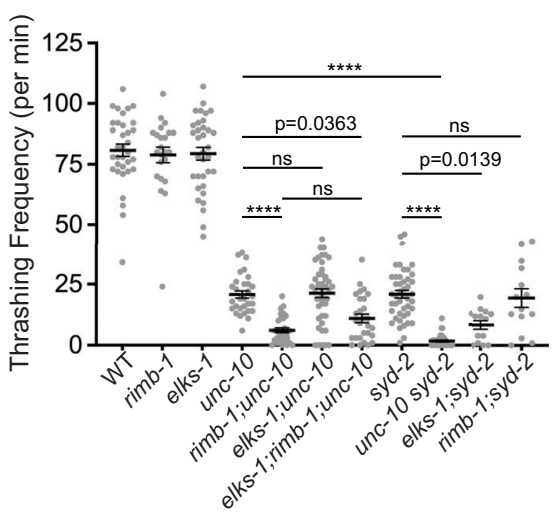

Figure 8. Effects of mutations in active zone component genes on locomotory function. The thrashing assay was used to assess the locomotory function of indicated mutants; $24 \mathrm{~h}$ post-L4 stage animals were assayed for $1 \mathrm{~min}$ after $5 \mathrm{~min}$ of acclimation to the liquid. The error bars represent SEM. One-way ANOVA with Tukey's post hoc analysis. ${ }^{* * *} p<0.0001$, ns, not significant.

double mutants but not elks-1(ok2762);unc-10(md1117) double mutants showed further decreased thrashing. We did not observe a significant difference between rimb-1(ce828);elks-1 (ok2762);unc-10(md1117) triple mutants and rimb-1(ce828); 

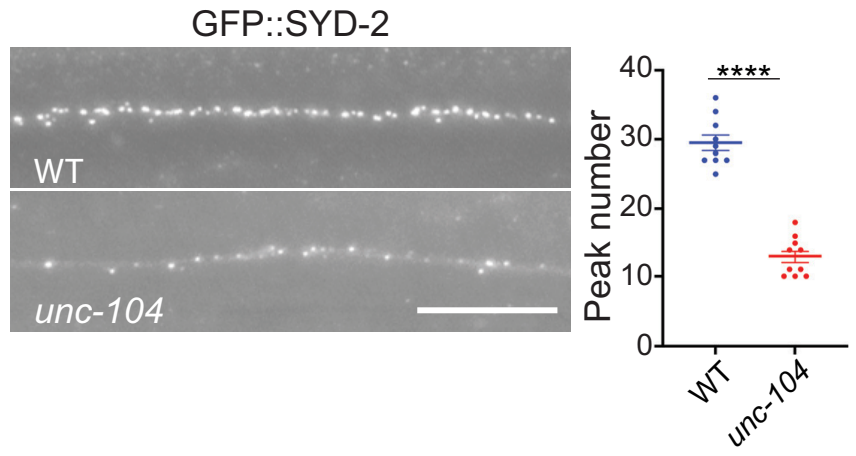

UNC-10::GFP
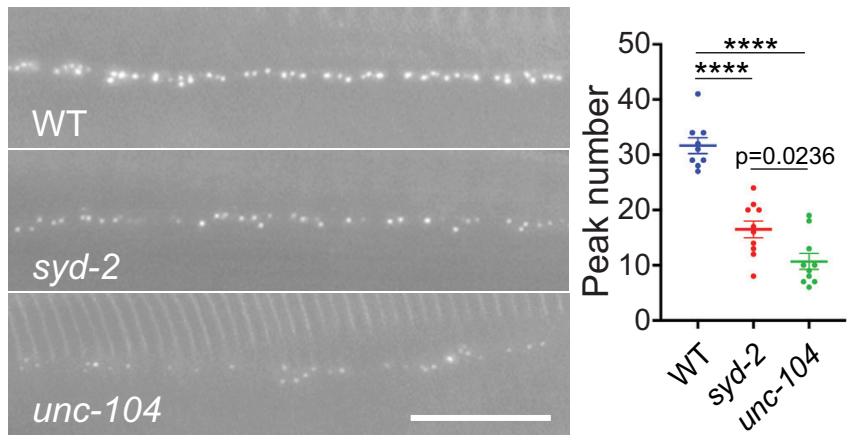
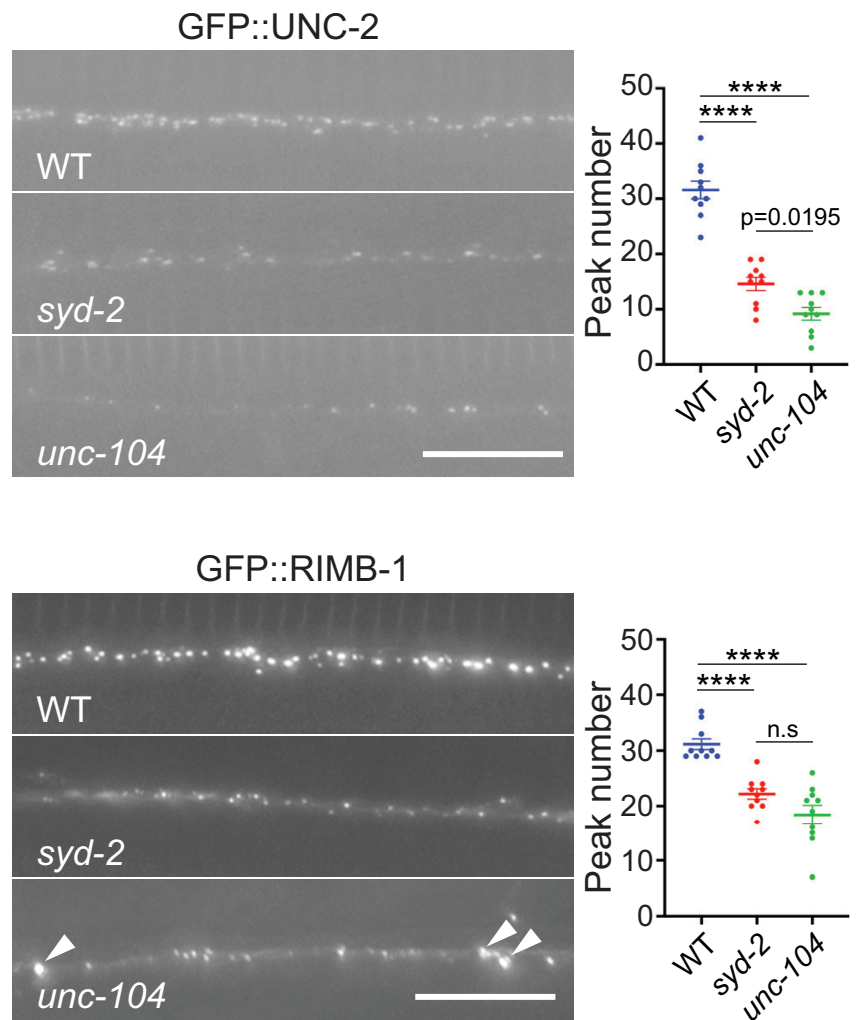
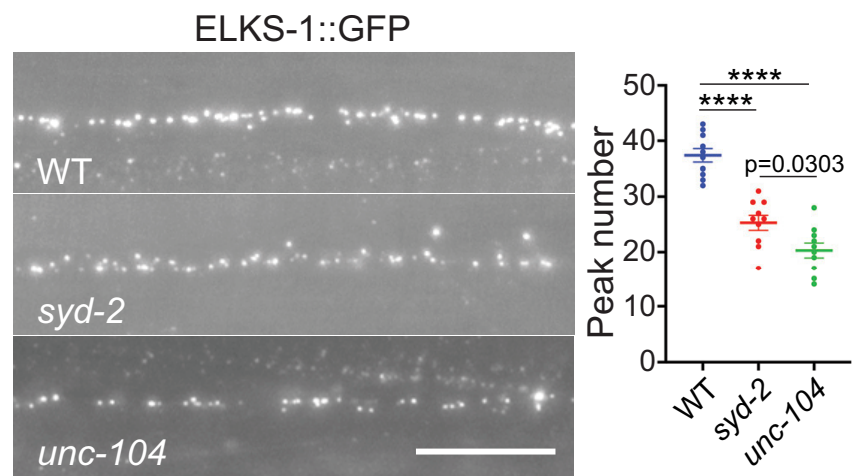

Figure 9. UNC-104/KIF1a is required for the presynaptic localization of UNC-2, UNC-10, RIMB-1, ELKS-1, and SYD-2. UNC-2, UNC-10, RIMB-1, and ELKS-1 in the dorsal nerve cords of wildtype, unc-104, and syd-2 mutant animals. SYD-2 in the dorsal nerve cords of wild-type and unc-104 mutant animals. Scale bar, $10 \mu \mathrm{m}$. The arrowheads indicate large aggregated RIMB-1 puncta. Error bars represent SEM. ${ }^{* * *} p<0.0001$, unpaired $t$ test for GFP::SYD-2 and one-way ANOVA with Tukey's post hoc analysis for the rest.

unc-10(md1117) double mutants, probably because both mutants were extremely lethargic in liquid. Likewise, reduced thrashing frequency of syd-2(ok217) mutants was further decreased by the elks-1(ok2762) but not rimb-1(ce828) mutation. Additionally, unc-10(md1117) syd-2(ok217) double mutants exhibited lower thrashing frequency than unc-10(md1117) or syd2(ok217) single mutant animals. Together, thrashing behavior of core active zone mutants correlates with UNC-2 channel abundance.

\section{UNC-104/KIF1A is required for axonal transport of SYD-2,} UNC-10, RIMB-1, ELKS-1, and UNC-2

In addition to active zone assembly/stability, SYD-2 has been reported to have a role in the transport of synaptic vesicles and active zone proteins (Wagner et al., 2009; Wu et al., 2013; Edwards et al., 2015). Thus, it could be argued that the role of SYD-2 in presynaptic UNC-2 localization can be entirely explained by its upstream role in the axonal transport of CAZ proteins. UNC-104/KIF1A is the major kinesin motor protein necessary for the transport of synaptic vesicles (Hall and Hedgecock, 1991; Okada et al., 1995). We examined whether UNC-104 is necessary for the synaptic localization of UNC-2, UNC-10, RIMB-1, ELKS-1, and SYD-2 in the dorsal nerve cord. Because our results indicate that SYD-2 plays a key role in the active zone localization of UNC-2, UNC-10, RIMB-1, and ELKS1, we first examined SYD-2 localization in a hypomorphic allele of unc-104(e1265) mutant known to severely impair vesicle transport while avoiding the lethality associated with unc-104 null mutants (Fig. 9). SYD-2 expression was significantly reduced in the dorsal nerve cord, indicating that axonal transport of SYD-2 is dependent on UNC-104. Because UNC-2, UNC-10, RIMB-1, and ELKS-1 are partially dependent on SYD-2 for active zone localization, synaptic expression was also expected to be disrupted in unc-104 mutant. Indeed, the dorsal nerve cord 


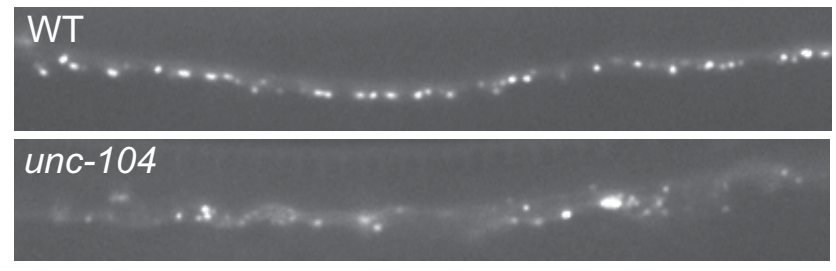

Movie 1. Time-lapse videos of endogenously GFP-tagged RIMB-1 in the dorsal nerve cords of wild-type and unc-104(e1265) mutant animals. [View online]

localization of all four proteins was disrupted in $u n c-104$ mutant (Fig. 9). In addition, the number of UNC-2, UNC-10, and ELKS1 puncta is consistently lower in unc-104 mutants than in syd-2 mutants. Unlike UNC-2, UNC-10, and ELKS-1, RIMB-1 puncta are highly variable in size and number and mobile in unc-104 mutants (Movie 1). Together, the localization of UNC-2, UNC10, RIMB-1, and ELKS-1 was more severely disrupted in unc-104 mutants than in the syd-2 mutant background. This is consistent with the observations that a fraction of UNC-10, RIMB-1, and ELKS-1 proteins can localize to presynaptic terminals independently of SYD-2, and this SYD-2-independent localization is dependent on UNC-104.

In summary, we have shown that UNC-2 is localized to active zones by both SYD-2-dependent and SYD2-independent mechanisms (Fig. 10). In the SYD-2-dependent mechanism, UNC-10, RIMB-1, and ELKS-1 are localized at the active zone through SYD-2, and these proteins in turn tether UNC-2 channels at active zones. In the SYD-2-independent mechanism, UNC-2 channels are localized to active zones by UNC-10 and ELKS-1.

\section{Discussion}

In this study we used CRISPR/Cas9 genome editing to label endogenous CaV2/UNC-2 and performed a genetic screen to identify genes that control UNC-2 trafficking and localization. In addition to previously identified genes known to affect UNC-2 trafficking and function, we isolated $u n c-10$ and syd-2, two genes encoding key active zone proteins. Consistent with previous findings observed in knock-out mice of all RIM isoforms (Kaeser et al., 2011), unc-10 null mutants exhibit a significant reduction of endogenous UNC-2 puncta at presynaptic active zones. Similarly, syd-2 mutants also exhibit profound loss of presynaptic UNC-2 puncta in neurons. SYD-2 is known to have a broad function in the localization and stabilization of other active zone proteins at presynaptic terminals (Zhen and Jin, 1999; Dai et al., 2006; Patel et al., 2006). Here, we show that in syd-2 mutants, presynaptic UNC-10, RIMB-1, and ELKS-1 puncta were all reduced but not eliminated. Our genetic analysis indicates that the recruitment and stabilization of UNC-10 and RIMB-1 at presynaptic terminals by SYD-2 are critical for presynaptic UNC-2 localization, whereas UNC-10 and ELKS-1 also contribute to presynaptic UNC-2 localization in a SYD-2-independent manner.

Our data show that UNC-10 is the most consequential effector among active zone proteins in localizing UNC-2 to presynaptic terminals. A similar reduction in the levels of $\mathrm{CaV} 2$ channels was observed in RIM1 and RIM2 double KO mice, suggesting this is a conserved process (Kaeser et al., 2011). Previous studies determined that $\mathrm{CaV} 2$ channels bind to the PDZ domain of RIM proteins via the C-terminal sequence (D/E-X-WC-COOH), which does not conform to classical PDZ binding motifs. Although the $C$. elegans $\mathrm{CaV} 2$ ortholog, UNC-2, is divergent from this motif sequence, it matches the classical PDZ binding motif (X- $\phi-\mathrm{X}-\phi-\mathrm{COOH})$, suggesting a coevolution of RIM and

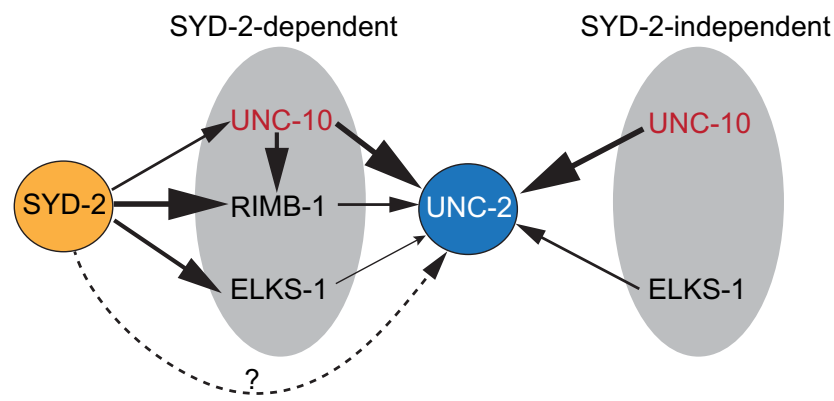

Figure 10. A genetic model for SYD-2-dependent and SYD-2-independent mechanisms of presynaptic UNC-2 localization. SYD-2 partly regulates presynaptic localization of UNC-10, RIMB-1, and ELKS-1. This regulation has some effects on UNC-2 localization mainly through UNC-10 and RIMB-1. However, as a fraction of UNC-10 localizes to presynaptic terminals even in the absence of SYD-2, UNC-10, with a minor role of ELKS-1, mediates UNC-2 localization independently of SYD-2.

$\mathrm{CaV} 2$ proteins. A previous study using isothermal calorimetry showed that the RIM PDZ domain stoichiometrically interacts with the C-terminal sequences of CaV2 channels in vitro (Kaeser et al., 2011). Consistent with that study, our data showed that copy numbers of UNC-2 and UNC-10 at presynaptic terminals are comparable.

Our genetic screen and analysis in C. elegans showed that SYD-2 is important for UNC-2 synaptic localization. The action of SYD-2 on presynaptic UNC-2 localization is more complicated than that of UNC-10 as SYD-2/Liprin- $\alpha$ has broad effects on active zone assembly and maintenance as well as axonal cargo transport (Zhen and Jin, 1999; Miller et al., 2005; Spangler et al., 2011; Zürner et al., 2011; Edwards et al., 2015; Böhme et al., 2016). A moderate reduction of UNC-10 puncta in syd-2 mutants cannot fully account for reduced UNC-2 puncta in syd2 mutants because the number of UNC-2 puncta is more severely reduced in syd-2 mutants than in unc-10 mutants. It is possible that SYD-2 acts on other active zone proteins that control UNC2 localization independently of UNC-10. Supporting this possibility, our data showed that SYD-2 partly acts on RIMB-1 to control UNC-2 localization.

SYD-2 was first identified as a critical mediator of synaptic vesicle cluster formation and active zone assembly in a C. elegans genetic screen (Zhen and Jin, 1999). Further genetic analyses with syd-2 mutants showed that SYD-2 recruits other major presynaptic proteins (Dai et al., 2006; Patel et al., 2006). In the Drosophila larval neuromuscular junction, Liprin- $\alpha /$ SYD-2 recruits $\mathrm{UNC13B}$ at the early stage of synaptogenesis, which serves as a template for the recruitment of Bruchpilot-RBP (RIM-binding protein) complexes to maturing synapses (Kaufmann et al., 2002; Astigarraga et al., 2010; Böhme et al., 2016). In mammals, knockout of Liprin- $\alpha 3$, the most prominent presynaptic Liprin- $\alpha$ protein in the brain, moderately reduces Munc13-1 and docked vesicles but does not cause dramatic reductions in other active zone proteins (Wong et al., 2018). Furthermore, Liprin- $\alpha 3$ is more mildly affected than those of other active zone proteins at synapses lacking RIM and ELKS (Wang et al., 2016) or RIM and RIM-BP (Acuna et al., 2016). These results may reflect that Liprin- $\alpha$ plays a role in early synaptogenesis independently of other active zone proteins. Alternatively, it is possible that as shown for other active zone proteins (Kaeser et al., 2011; Grauel et al., 2016), the absence of Liprin- $\alpha /$ SYD-2 does not affect the overall active zone organization. Indeed, our data show that SYD-2 is not absolutely required for active zone formation as key active zone proteins such as 
UNC-10, ELKS-1, and RIMB-1 form distinct puncta at active zones in syd-2 mutant animals, albeit at reduced levels. Moreover, syd-2 mutants retain significantly intact locomotory function (Fig. 8). Previous ultrastructural analyses of neuromuscular synapses showed that syd-2 mutant animals have fewer and smaller presynaptic-dense projections associated with fewer docked SVs than those of wild-type animals, indicating that synapse number is reduced but not eliminated in the absence of SYD-2 (Kittelmann et al., 2013).

Unexpectedly, the elks-1 mutation reduced UNC-2 localization in the absence of SYD-2. In C. elegans elks-1 mutants alone do not exhibit any obvious presynaptic phenotype (Deken et al., 2005). However, it was noted that ELKS-1 interacts with SYD-2 and is required for SYD-2 gain-of-function mutants to generate abnormally large dense projections (Kittelmann et al., 2013). A recent study has shown that SYD-2 and ELKS-1 undergo phase separation in vivo at the initial stage of synaptogenesis, and their fluidity is critical for efficient incorporation of active zone proteins (McDonald et al., 2020). Thus, although elks-1;syd-2 double mutants exhibit a dense projection structure comparable to syd-2 single mutants (Kittelmann et al., 2013), it is possible that the absence of both SYD-2 and ELKS-1 may cause a defect in the active zone assembly that prevents UNC-2 localization at active zones. On the other hand, mammalian ELKS/CAST binds to $\beta 4$ or $\alpha 1$ subunit of CaV2 (Kiyonaka et al., 2012), and Drosophila Bruchpilot/ELKS associates with Cacophony/CaV2 (Kittel et al., 2006). This raises the possibility that in the absence of SYD-2, ELKS-1 is one of the remaining links that directly supports UNC-2 localization via a physical interaction, and the absence of both SYD-2 and ELKS-1 severely compromises UNC-2 presynaptic localization.

One intriguing question is why UNC-10 and SYD-2 were not identified in prior C. elegans genetic studies with an integrated transgene overexpressing GFP-tagged UNC-2 (Saheki and Bargmann, 2009; Kushibiki et al., 2019). Presynaptic CaV2 channels are known to interact with several active zone proteins via direct and indirect interactions (Hibino et al., 2002; Fouquet et al., 2009; Kaeser et al., 2011; Kiyonaka et al., 2012; Tong et al., 2017; Luo et al., 2020). We speculate that in the absence of UNC10 or SYD-2, the residual overexpressed UNC-2 at presynaptic active zones, because of the weaker redundant interactions with other active zone proteins shown here, may not allow a detectable reduction in signal, whereas endogenously tagged UNC-2 does. These types of redundant interactions are common among active zone proteins. It is well documented that although the loss of individual active zone components generally causes at best mild synaptic deficits, simultaneous loss of two components results in severe defects in active zone organization (Acuna et al., 2016; Wang et al., 2016; Kushibiki et al., 2019).

On the other hand, there is compelling evidence that an increase in certain active zone proteins upregulates CaV2 channel density and synaptic transmission efficiency. For example, in Drosophila, it has been reported that presynaptic homeostatic plasticity accompanies presynaptic changes in RBP/RIM-BP, Bruchpilot/ELKS, and Cacophony/CaV2 (Müller et al., 2012, 2015; Böhme et al., 2019; Gratz et al., 2019). In future studies, it will be interesting to investigate whether and how altered synaptic activity reorganizes the active zone and modifies UNC-2 channel density and synaptic transmission in C. elegans.

\section{References}

Ackermann F, Waites CL, Garner CC (2015) Presynaptic active zones in invertebrates and vertebrates. EMBO Rep 16:923-938.
Acuna C, Liu X, Gonzalez A, Südhof TC (2015) RIM-BPs mediate tight coupling of action potentials to $\mathrm{Ca}(2+)$-triggered neurotransmitter release. Neuron 87:1234-1247.

Acuna C, Liu X, Südhof TC (2016) How to make an active zone: unexpected universal functional redundancy between RIMs and RIM-BPs. Neuron 91:792-807.

Arribere JA, Bell RT, Fu BX, Artiles KL, Hartman PS, Fire AZ (2014) Efficient marker-free recovery of custom genetic modifications with CRISPR/Cas9 in Caenorhabditis elegans. Genetics 198:837-846.

Astigarraga S, Hofmeyer K, Farajian R, Treisman JE (2010) Three Drosophila liprins interact to control synapse formation. J Neurosci 30:15358-15368.

Böhme MA, Beis C, Reddy-Alla S, Reynolds E, Mampell MM, Grasskamp AT, Lützkendorf J, Bergeron DD, Driller JH, Babikir H, Göttfert F, Robinson IM, O’Kane CJ, Hell SW, Wahl MC, Stelzl U, Loll B, Walter AM, Sigrist SJ (2016) Active zone scaffolds differentially accumulate Unc13 isoforms to tune $\mathrm{Ca}(2+)$ channel-vesicle coupling. Nat Neurosci 19:1311-1320.

Böhme MA, McCarthy AW, Grasskamp AT, Beuschel CB, Goel P, Jusyte M, Laber D, Huang S, Rey U, Petzoldt AG, Lehmann M, Göttfert F, Haghighi P, Hell SW, Owald D, Dickman D, Sigrist SJ, Walter AM (2019) Rapid active zone remodeling consolidates presynaptic potentiation. Nat Commun 10:1085.

Cheung TP, Choe JY, Richmond JE, Kim H (2020) BK channel density is regulated by endoplasmic reticulum associated degradation and influenced by the SKN-1A/NRF1 transcription factor. PLoS Genet 16: e1008829.

Dai Y, Taru H, Deken SL, Grill B, Ackley B, Nonet ML, Jin Y (2006) SYD-2 Liprin-alpha organizes presynaptic active zone formation through ELKS. Nat Neurosci 9:1479-1487.

Deken SL, Vincent R, Hadwiger G, Liu Q, Wang ZW, Nonet ML (2005) Redundant localization mechanisms of RIM and ELKS in Caenorhabditis elegans. J Neurosci 25:5975-5983.

Dolphin AC, Lee A (2020) Presynaptic calcium channels: specialized control of synaptic neurotransmitter release. Nat Rev Neurosci 21:213-229.

Dong W, Radulovic T, Goral RO, Thomas C, Suarez Montesinos M, Guerrero-Given D, Hagiwara A, Putzke T, Hida Y, Abe M, Sakimura K, Kamasawa N, Ohtsuka T, Young SM Jr (2018) CAST/ELKS proteins control voltage-gated $\mathrm{Ca}(2+)$ channel density and synaptic release probability at a mammalian central synapse. Cell reports 24:284-293.e6.

Edwards SL, Yorks RM, Morrison LM, Hoover CM, Miller KG (2015) Synapse-assembly proteins maintain synaptic vesicle cluster stability and regulate synaptic vesicle transport in Caenorhabditis elegans. Genetics 201:91-116.

Emperador-Melero J, Kaeser PS (2020) Assembly of the presynaptic active zone. Curr Opin Neurobiol 63:95-103.

Fouquet W, Owald D, Wichmann C, Mertel S, Depner H, Dyba M, Hallermann S, Kittel RJ, Eimer S, Sigrist SJ (2009) Maturation of active zone assembly by Drosophila Bruchpilot. J Cell Biol 186:129-145.

Gomez AM, Traunmüller L, Scheiffele P (2021) Neurexins: molecular codes for shaping neuronal synapses. Nat Rev Neurosci 22:137-151.

Gratz SJ, Goel P, Bruckner JJ, Hernandez RX, Khateeb K, Macleod GT, Dickman D, O'Connor-Giles KM (2019) Endogenous tagging reveals differential regulation of $\mathrm{Ca}(2+)$ channels at single active zones during presynaptic homeostatic potentiation and depression. J Neurosci 39:2416-2429.

Grauel MK, Maglione M, Reddy-Alla S, Willmes CG, Brockmann MM, Trimbuch T, Rosenmund T, Pangalos M, Vardar G, Stumpf A, Walter AM, Rost BR, Eickholt BJ, Haucke V, Schmitz D, Sigrist SJ, Rosenmund C (2016) RIM-binding protein 2 regulates release probability by fine-tuning calcium channel localization at murine hippocampal synapses. Proc Natl Acad Sci U S A 113:11615-11620.

Hall DH, Hedgecock EM (1991) Kinesin-related gene unc-104 is required for axonal transport of synaptic vesicles in C. elegans. Cell 65:837-847.

Held RG, Liu C, Kaeser PS (2016) ELKS controls the pool of readily releasable vesicles at excitatory synapses through its $\mathrm{N}$-terminal coiled-coil domains. Elife 5:e14862.

Hibino H, Pironkova R, Onwumere O, Vologodskaia M, Hudspeth AJ, Lesage F (2002) RIM binding proteins (RBPs) couple Rab3-interacting molecules (RIMs) to voltage-gated $\mathrm{Ca}(2+)$ channels. Neuron 34:411423.

Holderith N, Lorincz A, Katona G, Rózsa B, Kulik A, Watanabe M, Nusser Z (2012) Release probability of hippocampal glutamatergic terminals scales with the size of the active zone. Nat Neurosci 15:988-997. 
Huang YC, Pirri JK, Rayes D, Gao S, Mulcahy B, Grant J, Saheki Y, Francis MM, Zhen M, Alkema MJ (2019) Gain-of-function mutations in the UNC-2/CaV2a channel lead to excitation-dominant synaptic transmission in Caenorhabditis elegans. Elife 8:e45905.

Jin Y, Garner CC (2008) Molecular mechanisms of presynaptic differentiation. Annu Rev Cell Dev Biol 24:237-262.

Kaeser PS, Deng L, Wang Y, Dulubova I, Liu X, Rizo J, Südhof TC (2011) RIM proteins tether $\mathrm{Ca}(2+)$ channels to presynaptic active zones via a direct PDZ-domain interaction. Cell 144:282-295.

Kaufmann N, DeProto J, Ranjan R, Wan H, Van Vactor D (2002) Drosophila liprin-alpha and the receptor phosphatase Dlar control synapse morphogenesis. Neuron 34:27-38.

Kittel RJ, Wichmann C, Rasse TM, Fouquet W, Schmidt M, Schmid A, Wagh DA, Pawlu C, Kellner RR, Willig KI, Hell SW, Buchner E, Heckmann M, Sigrist SJ (2006) Bruchpilot promotes active zone assembly, Ca2 + channel clustering, and vesicle release. Science 312:1051-1054.

Kittelmann M, Hegermann J, Goncharov A, Taru H, Ellisman MH, Richmond JE, Jin Y, Eimer S (2013) Liprin- $\alpha$ /SYD-2 determines the size of dense projections in presynaptic active zones in C. elegans. J Cell Biol 203:849-863.

Kiyonaka S, Nakajima H, Takada Y, Hida Y, Yoshioka T, Hagiwara A, Kitajima I, Mori Y, Ohtsuka T (2012) Physical and functional interaction of the active zone protein CAST/ERC2 and the $\beta$-subunit of the voltagedependent $\mathrm{Ca}(2+)$ channel. J Biochem 152:149-159.

Ko J, Na M, Kim S, Lee JR, Kim E (2003) Interaction of the ERC family of RIM-binding proteins with the liprin-alpha family of multidomain proteins. J Biol Chem 278:42377-42385.

Kurshan PT, Merrill SA, Dong Y, Ding C, Hammarlund M, Bai J, Jorgensen EM, Shen K (2018) $\gamma$-neurexin and frizzled mediate parallel synapse assembly pathways antagonized by receptor endocytosis. Neuron 100:150-166.e4.

Kushibiki Y, Suzuki T, Jin Y, Taru H (2019) RIMB-1/RIM-Binding Protein and UNC-10/RIM redundantly regulate presynaptic localization of the voltagegated calcium channel in Caenorhabditis elegans. J Neurosci 39:8617-8631.

Liu C, Bickford LS, Held RG, Nyitrai H, Sudhof TC, Kaeser PS (2014) The active zone protein family ELKS supports Ca2 + influx at nerve terminals of inhibitory hippocampal neurons. J Neurosci 34:12289-12303.

Lübbert M, Goral RO, Keine C, Thomas C, Guerrero-Given D, Putzke T, Satterfield R, Kamasawa N, Young SM Jr (2019) CaV2.1 $\alpha 1$ subunit expression regulates presynaptic CaV2.1 abundance and synaptic strength at a central synapse. Neuron 101:260-273.e6.

Luo F, Sclip A, Jiang M, Südhof TC (2020) Neurexins cluster Ca(2+) channels within the presynaptic active zone. EMBO J 39:e103208.

Mahoney TR, Luo S, Nonet ML (2006) Analysis of synaptic transmission in Caenorhabditis elegans using an aldicarb-sensitivity assay. Nat Protoc 1:1772-1777.

McDonald NA, Fetter RD, Shen K (2020) Assembly of synaptic active zones requires phase separation of scaffold molecules. Nature 588:454-458.

Miller KE, DeProto J, Kaufmann N, Patel BN, Duckworth A, Van Vactor D (2005) Direct observation demonstrates that Liprin-alpha is required for trafficking of synaptic vesicles. Curr Biol 15:684-689.

Müller M, Liu KS, Sigrist SJ, Davis GW (2012) RIM controls homeostatic plasticity through modulation of the readily-releasable vesicle pool. J Neurosci 32:16574-16585.

Müller M, Genç Ö, Davis GW (2015) RIM-binding protein links synaptic homeostasis to the stabilization and replenishment of high release probability vesicles. Neuron 85:1056-1069.

Nyitrai H, Wang SSH, Kaeser PS (2020) ELKS1 Captures Rab6-marked vesicular cargo in presynaptic nerve terminals. Cell Rep 31:107712.

Oh KH, Kim H (2017) Aldicarb-induced paralysis assay to determine defects in synaptic transmission in Caenorhabditis elegans. Bio Protoc 7:e2400.

Oh KH, Abraham LS, Gegg C, Silvestri C, Huang YC, Alkema MJ, Furst J, Raicu D, Kim H (2015) Presynaptic BK channel localization is dependent on the hierarchical organization of alpha-catulin and dystrobrevin and fine-tuned by CaV2 calcium channels. BMC Neurosci 16:26.

Oh KH, Haney JJ, Wang X, Chuang CF, Richmond JE, Kim H (2017) ERG28 controls BK channel trafficking in the ER to regulate synaptic function and alcohol response in C. elegans. Elife 6:e24733.

Oh KH, Sheoran S, Richmond JE, Kim H (2020) Alcohol induces mitochondrial fragmentation and stress responses to maintain normal muscle function in Caenorhabditis elegans. FASEB J 34:8204-8216.
Ohtsuka T, Takao-Rikitsu E, Inoue E, Inoue M, Takeuchi M, Matsubara K, Deguchi-Tawarada M, Satoh K, Morimoto K, Nakanishi H, Takai Y (2002) Cast: a novel protein of the cytomatrix at the active zone of synapses that forms a ternary complex with RIM1 and munc13-1. J Cell Biol 158:577-590.

Okada Y, Yamazaki H, Sekine-Aizawa Y, Hirokawa N (1995) The neuronspecific kinesin superfamily protein KIF1A is a unique monomeric motor for anterograde axonal transport of synaptic vesicle precursors. Cell 81:769-780.

Patel MR, Lehrman EK, Poon VY, Crump JG, Zhen M, Bargmann CI, Shen K (2006) Hierarchical assembly of presynaptic components in defined C. elegans synapses. Nat Neurosci 9:1488-1498.

Petzoldt AG, Götz TWB, Driller JH, Lützkendorf J, Reddy-Alla S, MatkovicRachid T, Liu S, Knoche E, Mertel S, Ugorets V, Lehmann M, Ramesh N, Beuschel CB, Kuropka B, Freund C, Stelzl U, Loll B, Liu F, Wahl MC, Sigrist SJ (2020) RIM-binding protein couples synaptic vesicle recruitment to release sites. J Cell Biol 219:e201902059.

Pierce-Shimomura JT, Chen BL, Mun JJ, Ho R, Sarkis R, McIntire SL (2008) Genetic analysis of crawling and swimming locomotory patterns in C. elegans. Proc Natl Acad Sci U S A 105:20982-20987.

Radulovic T, Dong W, Goral RO, Thomas CI, Veeraraghavan P, Montesinos MS, Guerrero-Given D, Goff K, Lübbert M, Kamasawa N, Ohtsuka T, Young SM Jr (2020) Presynaptic development is controlled by the core active zone proteins CAST/ELKS. J Physiol 598:2431-2452.

Richmond JE (2006) Electrophysiological recordings from the neuromuscular junction of C. elegans. WormBook: the online review of. Wormbook 2006:1-8.

Saheki Y, Bargmann CI (2009) Presynaptic CaV2 calcium channel traffic requires CALF-1 and the alpha(2)delta subunit UNC-36. Nat Neurosci 12:1257-1265.

Spangler SA, Jaarsma D, De Graaff E, Wulf PS, Akhmanova A, Hoogenraad CC (2011) Differential expression of liprin- $\alpha$ family proteins in the brain suggests functional diversification. J Comp Neurol 519:3040-3060.

Südhof TC (2012) The presynaptic active zone. Neuron 75:11-25.

Taru H, Jin Y (2011) The Liprin homology domain is essential for the homomeric interaction of SYD-2/Liprin- $\alpha$ protein in presynaptic assembly. J Neurosci 31:16261-16268.

Tong XJ, Lopez-Soto EJ, Li L, Liu H, Nedelcu D, Lipscombe D, Hu Z, Kaplan JM (2017) Retrograde synaptic inhibition is mediated by $\alpha$-neurexin binding to the $\alpha 2 \delta$ subunits of $\mathrm{N}$-type calcium channels. Neuron 95:326-340. e325.

van den Maagdenberg AM, Haan J, Terwindt GM, Ferrari MD (2007) Migraine: gene mutations and functional consequences. Curr Opin Neurol 20:299-305.

Wagner OI, Esposito A, Kohler B, Chen CW, Shen CP, Wu GH, Butkevich E, Mandalapu S, Wenzel D, Wouters FS, Klopfenstein DR (2009) Synaptic scaffolding protein SYD-2 clusters and activates kinesin-3 UNC-104 in C. elegans. Proc Natl Acad Sci U S A 106:19605-19610.

Wang SSH, Held RG, Wong MY, Liu C, Karakhanyan A, Kaeser PS (2016) Fusion competent synaptic vesicles persist upon active zone disruption and loss of vesicle docking. Neuron 91:777-791.

Wang Y, Liu X, Biederer T, Sudhof TC (2002) A family of RIM-binding proteins regulated by alternative splicing: implications for the genesis of synaptic active zones. Proc Natl Acad Sci U S A 99:14464-14469.

Wong MY, Liu C, Wang SSH, Roquas ACF, Fowler SC, Kaeser PS (2018) Liprin- $\alpha 3$ controls vesicle docking and exocytosis at the active zone of hippocampal synapses. Proc Natl Acad Sci U S A 115:2234-2239.

Wu YE, Huo L, Maeder CI, Feng W, Shen K (2013) The balance between capture and dissociation of presynaptic proteins controls the spatial distribution of synapses. Neuron 78:994-1011.

Zhang W, Rohlmann A, Sargsyan V, Aramuni G, Hammer RE, Südhof TC, Missler M (2005) Extracellular domains of alpha-neurexins participate in regulating synaptic transmission by selectively affecting $\mathrm{N}$ - and P/Q-type Ca2 + channels. J Neurosci 25:4330-4342.

Zhen M, Jin Y (1999) The liprin protein SYD-2 regulates the differentiation of presynaptic termini in C. elegans. Nature 401:371-375.

Zürner M, Mittelstaedt T, tom Dieck S, Becker A, Schoch S (2011) Analyses of the spatiotemporal expression and subcellular localization of liprin- $\alpha$ proteins. J Comp Neurol 519:3019-3039. 